\title{
From Individual to Collective Pinning: Effect of Long-range Elastic Interactions
}

\author{
Anne Tanguy ${ }^{+}$, Matthieu Gounelle ${ }^{+, *}$ \\ + Laboratoire de Physique et de Mécanique des Milieux Hétérogènes, \\ URA CNRS n $n^{\circ} 85 \%$, \\ Ecole Supérieure de Physique et Chimie Industrielles de Paris, \\ 10, rue Vauquelin, 75231 Paris Cedex 05, France. \\ * Permanent Address: \\ Centre de Spectrométrie de Nucléaire et de Spectrométrie de Masse \\ Bâtiment 104, 91485 Orsay Campus, France.
}

Stéphane Roux

Laboratoire Surface du Verre et Interfaces, Unité Mixte de Recherche CNRS/St-Gobain, 39 Quai Lucien Lefranc, F-93303 Aubervilliers Cedex, France.

(August 13, 2018)

\begin{abstract}
We study the effect of long-range elastic interactions in the dynamical behavior of an elastic chain driven quasi-statically in a quenched random pinning potential. This is a generic situation occuring in solid friction, crack propagation, wetting front motion, ... In the strong pinning limit, the dynamic of the chain is controlled by individual instabilities of each site of the chain. Longrange correlations in the displacement field and in the force field develop progressively. The system self-organizes to a steady state where the propagation of the instabilities is described by scaling laws with characteristic critical exponents. These exponents are numerically estimated through the analysis of the spatio-temporal correlation in the activity map. Tuning the exponent $\alpha$ of the algebraic decay of the elastic interaction with the distance is shown to give rise to three regimes: a Mean-Field (MF) regime valid for $\alpha<1$ (very slow decay), a Laplacian (L) regime for $\alpha>3$ (rapid decay of interactions), and an intermediate regime $1<\alpha<3$ where the critical exponents interpolate continuously between the MF and L limit cases. The latter regime is shown to display, in the range $1<\alpha<2$, a mean-field type character only for time-correlations but not for space. The effect of the driving mode on the avalanche statistics is also analyzed.
\end{abstract}

PACS numbers: 05.40+j, 74.60.Ge, 64.60.Ht, 64.60.LxSelf

\section{INTRODUCTION}

Multistability of elastic media in a pinning potential is responsible for the complex dissipative behavior observed in various physical situations like motion of vortices in type II superconductorst, dynamics of a ferromagnetic domain wall driven by an external magnetic field thfough a random mediumb, Charge Density Waves (CDW 3 , roughening of crack fronts in fracture 4 , or solid friction 5 .

In the latter case, the dynamic of the slider can be reduced to that of the asperities at the surfaces of the solid 6 . The role of the elastic body is to mediate the interactions between asperities and with the pulling machine. It has been shown recently that the competition between the elastic restoring force due to the bulk and the non linear pinning force, due to the contact between asperities of different solids, gives rise to multiple stable equilibrium positions when the pinning forces are sufficiently strong, or when the system is sufficiently large. This multistability is responsible for hysteretic behaviour of asperities, when they are driven quasi-statically over the set of pinning centers belonging to the surface of the other solid. Dissipation arises from this hysteretic behaviour and it takes place in the solid, which plays as well the role of thermal bath. In the case of spreading of a partially wetting liquid on a heterogeneous pland, the evolution of the contact line depends on the competition between a "pinning force" due to surface heterogeneities, and an elastic restoring force resulting from surface tension. In fact, a distortion of wavelength $\lambda$ of the three-phase front modifies the liquid-vapor interface over the same distance away from the wall, thus resulting, after integration over the whole liquid-vapor surface, in a effective elastic restoring force

$$
f(x)=-k \int \frac{u(x)-u\left(x_{1}\right)}{\left|x-x_{1}\right|^{\alpha}} d x_{1}
$$

with $\alpha=2$. The local capillary force is a non local function of the entire contact lipe position. The same effect holds for crack propagations, where the stress intensity factor exhibits a non local dependence on the front geometry, with $\alpha=2$ as well. In the case of solid friction, the couplings due to the three dimensional elasticity are scale invariant and their translationaly invariant part has the same form (11), with $\alpha=2 D-1$ for a D-dimensional 
lattice of asperities coupled via a three-dimensional solid (thus $\alpha=1$ for $D=1$ and $\alpha=3$ for $D=2$, as can - ee checked by Fourier transforming the usual relation $\$ 1$ ). Expression (1) is also compatible with dipolar interactions $(\alpha=3)$ in the case of ferromagnetic domain wall $(\mathrm{D}=2)$, and we will see that it also takes into account the usual Laplacian couplings of vortex lattices, charge density waves ...

The effect of the long range coupling on the dynamics of the system has been little studied since each event involves a large part of the system and can generally not be solved perturbatively. However, it is known 11 to have a strong influence on the behavior of the system as well as on its stability properties. Here, we focus on the behaviour of an elastic line $(D=1)$ with long range interactions, driven quasi-statically on a substrate with quenched disorder, and in the strong pinning limit. We want to show the effect of the long-range elastic couplings on the fluctuations accompanying the average behaviour of the line.

By analogy with friction, we will call "asperity" each point of discretization of the elastic line. In general, two situations may hold: if the couplings are small in comparison to the distortions of the pinning potential, then the motion of the chain is controlled by the motion of each asperity. This is the strong pinning limit. In this case, when the local stiffness is sufficiently weak, the stable local equilibrium position of an asperity may disappear, and the "unstable" asperity advances suddenly to the next equilibrium position. In contrast, in the weak pinning limit, when the couplings are sufficiently strong, the line behaves in a first approximation as a whole. In such a case, a separation in domains may occur for a sufficiently large system sizel. Thus a coarse-graining of the system at large enough scales leads to a simple strongpinning regime. The latter case thus appears to be the possible relevant limit in the thermodynamic limit of a infinite sized system.

In this paper, we investigate the effect of the range of elastic interactions in the strong pinning case, where the fluctuating dynamics of the system results from the deterministic propagation of local instabilities. More precisely, we focus our attention on the interactions between asperities and we consider only the elastic displacement field components whose wavelength is greater than the distance between "asperities". This approach is complementary to that of considering individual jumps without couplingst. We show how the nature of the elastic interactions allows to interpolate between a "delocalized" (or mean field) regime and a "local" regime analogous to the Laplacian case. The driving mode is shown to affect the velocity of propagation of the instabilities and the avalanche statistics.

In the first part of the paper, we propose a discrete model of the dynamics which will be compared to the continuous one. Then we study in a systematic way the dependence on the decay of elastic interactions, $\alpha$ in Eq.(1), of the propagation of instabilities, of the or- ganization of the dynamic and of the kinetic roughening. The last section is devoted to a discussion about the universality of our model, and on the uniformization due to elastic interactions.

Let us recall briefly that $\alpha=1$ for an elastic onedimensional chain with "three-dimensional" couplings, but $\alpha=-2$ for an elastic string with bending glasticity $[0$, $\alpha=2$ for fracture dynamics in infinite splids 9 or wetting experiments in a free surface geometry $d$, and $\alpha=0$ in propagating capillary fronts due to the fluid flow behind the front 13 .

It is important to note that, for a given physical system, $\alpha$ may be changed experimentally by changing the geometry of the apparatus. This is, for example, the case of wetting, where $\alpha=2$ in a free surface geometry, but where the couplings are Laplacian at large scales in a Hele-Shaw cell (at vanishing capillary number) 14.

\section{THE MODEL}

Our aim is to single out the effect of interactions in the propagation of instabilities in an elastic chain $(D=1)$ driven quasi-statically along its transversal direction over a rough surface. We consider here a discrete one dimensional elastic chain of size $L$. The chain of size $L$ is discretized into $L / d$ blocks. $d$ represents the distance between asperities in the strong pinning limit, and more generally the distance between "sites". In the case of wetting for example, it will be at least on the order of the distance between pinning centers. It is not possible to have access to information below $d$ in our model; $d$ is the lower cut-off of our model and $L$ is the upper cut-off.

We call $\mathbf{x}$ the current site which is the position of the site evaluated in the non deformed geometry. The position of the site in the deformed geometry is $\mathbf{x}^{\prime}=\mathbf{x}+\mathbf{u}(\mathbf{x})$, where $\mathbf{u}(\mathbf{x})$ is the displacement field of the chain in the laboratory frame. We will refer as well to $\mathbf{u}(\mathbf{x})$ as the difference in the actual position $\mathbf{x}^{\prime}$ of site $\mathbf{x}$ to any homogeneous motion of the undeformed geometry. We consider only the large wavelength components of the displacement field $(\lambda>d)$, thus $\mathbf{u}(\mathbf{x})$ corresponds to the displacement averaged over a cell of size $d^{D}$. In the following, we take account only for the displacement in the direction of pulling, $\mathbf{j}$, transversal to the chain, and we neglect the displacement along the chain. The displacement field is a scalar and it will be noted $u(x)$, as shown in Fig. (1).

Each site of the chain is subjected to a driving force, a random pinning force (from the interface) and a long range elastic force describing the coupling to the other sites of the chain. The continuous equation of motion for this system could thus be written in the overdamped limit

$$
\gamma \frac{\partial u(x, t)}{\partial t}=\left[F_{e x t}(t)+\eta(x, u(x))\right.
$$




$$
\left.-k \int \frac{u(x)-u\left(x_{1}\right)}{\left|x-x_{1}\right|^{\alpha}} d x_{1}\right]
$$

where $F_{\text {ext }}$ stands for the driving force and $\eta(x, u(x))$ for the quenched pinning force. The left-hand side of the equation contains a phenomenological damping term.

The long range elastic force has been already introduced in the previous part of this article. Let us remark that it may be described by three fundamental properties: (1) it is linear in $u(x)$ (linear elasticity), (2) it is scale invariant (despite the lower cut-off $d$ and the upper cut-off $L$ ), (3) and it is "translationaly invariant". This latter property is due to the fact that we call "coupling forces" only internal forces. These forces do not contribute to the average over all the medium. This is compatible with the fact that the elastic energy of the chain (in absence of pinning) is translationaly invariant. Any uniform displacement of the chain does contribute to an average restoring force which has to be compensated at equilibrium by a pinning forfe; but contrary to the definition used by C.Caroli et al.5, it does not contribute to the elastic couplings. These couplings have an infinite range due to their algebraic decay. For the sake of simplicity, we will refer to $\alpha$ as the range of the interaction. As $\alpha$ increases, the interaction tends to be concentrated dominantly on the nearest neighbors. On the contrary for a small $\alpha$, the interaction tends to be more evenly distributed over the system. We will see later how to adjust the external driving force to take account for the average dynamic of the system. The quenched pinning force, $\eta$, represents the interaction between the heterogeneous surface and the elastic chain. In case of solid friction, it may have various physical origins (Hertzian contact between asperities of different surfaces, adhesion ...) but it is always non linear. Moreover, a large scale description of the contact problem supposes that the basic interaction is already the combination of multiple elementary precesses, giving rise to discontinuities and multivaluation 5 . At a sufficiently coarse-grained level, the effective force of the interaction will loose its continuity. In order to be able to describe the possible fixed point of a renormalized description of the surface interactions, we choose to use a discontinuous pinning force, described only by its statistical properties. This force is quenched and random. We will restrict ourself in the following to white noise. Moreover, we anticipate that the fluctuations of the amplitude of the pinning force are small when the slider moves forward, and not sufficiently high to allow backward motion of the slider. This is also the case when spatial distortions of the pinning forces are strong. In this case, it is still possible to use the expression (2) but with the reservation that only the positive part of the r.h.s. is considered. Thus, the system is supposed to move only forward and the pinning force may be described for each site $x$ only by a position coordinate $i(x)$ and a threshold value $\eta_{i(x)}(x)$ as shown in Fig. (1.b). In such a manner, our model contains the main features of the dynamics of an elastic chain, driven over a pinning surface in the strong pin- ning limit. It is also believed to capture the large scale behavior of any pinning potential. But, considering deliberately the strong pinning regime, we cannot address the interesting question of the cross-over scale from weak to strong pinning.

We are interested in the quasi-static limit of the motion of the chain. In this case, the damping is supposed to be sufficiently strong to allow each site to reach instantaneously its local equilibrium position. In the quasi-static approximation, it is well known that, in the stationary regime and in the strong pinning limit, the main contribution to the global displacement is due to hysteretic jumps of each element of the chain, resulting from the multistability of the site and of the delay ruld 17 . For a given external driving, the initiation of a jump is a local instability and thus the dynamics cannot be controlled simply by that of the center of mass. To avoid the explicit introduction of the dynamic of the system (with inertia and viscous damping), and thus the necessity to integrate the equation of motion, we assume the following conditions. First, the system evolves from one local equilibrium position to the next closest one. Second, we monitor the driving force $F_{\text {ext }}(t)$ actively so that we always maintain the system at the edge of stability. More precisely, $F_{\text {ext }}(t)$ is adjusted so that only one mode is metastable (only one site jumps at each time step). Let us emphasize that in contrast to many approaches based on continuum equations as Eq. (2), we do not consider a constant force but rather a constant but infinitesimal mean velocity. In solid friction, it corresponds to a uniform average imposed displacement $\mathbf{v}$ on the top side of the solid. In wetting experiments, it will correspond to motion at an imposed slow rate of flow. Alternatively, $\mathbf{v}$ may be deduced from a motion where a force is imposed on the top side of the solid (given pressure in wetting experiments). $\mathbf{v}$ is related, via shear of the solid, to the average position of asperities of the chain. When the displacement is imposed, the discrete version of our model, as studied below, belongs to the class of extremal mpdels (such as invasion percolation 19 , fracture model 20 or more recently introduced growth model 21 ). With the second assumption, the motion will consist in a series of equilibrium points, and the equation of motion does not have to be integrated. Under the assumption of the over-damped nature of the motion, we will discuss a possible extrapolation of the obtained results to different driving modes. This discussion will allow us to relate the avalanche statistics to the observable intermittency of the motion of the chain.

The above introduced model is discretized to allow for numerical simulations. The string is described as a one-dimensional, periodic array of sites. The unit length along the chain is given by the distance between the sites of the chain, and the unit time by the time step $\delta t$ ellapsed between subsequent jumps. Our "time" count indeed the number of jumps (that is the main travelled distance). When one site depins from one asperity, it jumps by a distance $\delta u$, whose maximum value gives the unit 
length in the direction of propagation. The pinning potential is described by a set of discrete centers, with a random spatial distribution. We assume that the amplitude of each pinning force is identical throughout the medium. Alternatively, we could have chosen a uniform distribution (on a regular lattice) but with a random magnitude. We checked that these two variants (and their combination) give the same results, as soon as some randomness is introduced. For a periodic array of pinning centers with the same amplitude, as could have been guessed, the chain motion finally locks on a periodic motion of little interest.

The model runs as follows. We start with a uniform flat front, $u(x)=0$, for each cell. We suppose that these positions correspond to equilibrium positions in absence of external driving force (that is for each $x, \eta(x, 0)=0$ ). The external driving force $F_{\text {ext }}$ is applied progressively. The elastic displacement is negligible, thus only the pinning force increases first $\left(\eta(x, u(x, t))=-F_{\text {ext }}(t)\right)$. When the load is sufficiently high, one cell overcomes its threshold, and jumps. The maximum load is in this case

$$
F_{\text {ext }}=\min _{x}\left(-\eta_{0}(x)\right) .
$$

The cell $x^{*}$ which overcomes its threshold then jumps to the next basin $i\left(x^{*}\right)$, thus

$$
u\left(x^{*}\right) \rightarrow u\left(x^{*}\right)+\delta u
$$

During the jump, the external driving force is constant. The linearity of the problem allows us to calculate the new elastic couplings. The elastic forces along the chain are modified to

$$
f(x) \rightarrow f(x)+\delta u . G\left(x, x^{*}\right)
$$

where $G(x, y)$ results from the interaction kernel discussed above. The external driving force is then reduced to zero in order to prevent another jump, and the external load may be increased until

$$
F_{e x t}=\min _{x}\left(-\eta_{i(x)}(x)-f(x)\right)
$$

in order to allow the next jump. The discrete interaction kernel is chosen as the simplest expression which captures the required periodic boundary condition, and follows the power-law decay of Eq. (11):

$$
\begin{aligned}
G\left(x, x^{*}\right) & =\left[\sin \left(\pi \frac{\left|x-x^{*}\right|}{L}\right)\right]^{-\alpha} \quad \text { for } x \neq x^{*} \\
G\left(x^{*}, x^{*}\right) & =-\Sigma_{x \neq x^{*}} G\left(x, x^{*}\right)
\end{aligned}
$$

In the particular case of $\alpha=2$, such an expression (3) is the exact result of the summation of interactions for a periodic chain.

This describes one elementary step of the model. We start from a uniform flat front and run a long sequence of such steps until the system reaches a statistically stationary state. Condition Eq. (4) stands for the average conservation of forces along the chain. We used
$G\left(x^{*}, x^{*}\right) \equiv 1$, thus the stiffness for neighboring sites is unity. This sets the scale for forces. The jump size $\delta u$ thus reflects simply the distance between asperities on the track 17. The dynamic is "extremal" in the sense that only the site submitted to the maximum force $f\left(x^{*}\right)$ advances at each time step.

Fig.2 shows examples of the space time distribution of "active sites" $x^{*}$ in the medium for various couplings, $\alpha$. Fig. 3 shows the time evolution of the displacement front along the chain for the same values of $\alpha$. It appears clearly from these figures that the system organizes in a statistically stationary state, and that for large $\alpha$ (local couplings), the evolution is spatially inhomogeneous.

We compared several statistical distributions for $\delta u$ e.g. uniform in the interval $[a, 1]$. Our results at sufficiently large times and scales did not display any dependence on $a$ as long as $a \neq 1$. As noted above when $a=1$, the pinning centers are periodically distributed, with the same strength, and the chain motion ends up being periodic with no interesting features.

Similar models have already been studied 8 in the case $\alpha=2$ and in the limit as $\alpha \vec{\infty}$ which is the discrete version of the Laplacian one 22.23 and we will refer to them in the following. Similarly, the case $\alpha=0$ corresponds clearly to a mean-field situation and it admits a simple analytical solution which is quite similar to the situation solved by Flyvbjerg et ale4.

As far as one is interested only in the effect of the long range elastic interactions, it appears convenient to consider $\alpha$ as a continuous parameter of the model, albeit in most physical cases, only integer values can be found.

\section{KINETIC ROUGHENING}

Starting with an uncorrelated distribution of forces, the system organizes, after a transient, into a highly correlated statistically stationary state. One manifestation of these long range correlations is the roughening of the displacement front of the chain. The roughness of the chain is characterized by the scaling of the correlation function

$$
\left\langle(u(x+\vartheta)-u(\vartheta))^{2}\right\rangle_{\vartheta} \propto x^{2 \zeta}
$$

or in Fourier space

$$
\left\langle|\tilde{u}(k)|^{2}\right\rangle \propto k^{-1-2 \zeta}
$$

Such a power-law behavior of the power spectrum of the front position is shown on Fig. A for $\alpha=2$. $\zeta$ is called the "roughness exponent" of the front. From Fig. (1) we determine $\zeta=0.35 \pm 0.02$ in good agreement with a previgus determination of this exponent by Schmittbuhl et all.

As can be seen in Fig. (5), $\zeta$ depends on $\alpha$. The larger $\alpha$, the larger the roughness exponent, i.e. the more persistent the front fluctuation. Note that for large $\alpha$, the 
roughness exponent exceeds one, and thus Eq. (5) is inappropriate. Either one should use such a correlation function computed on the slope of the front (and measures $\zeta-1$ ), or revert to the spectral method for determining the roughness exponent. For $\alpha=3$, we obtain $\zeta \approx 1.2$ again consistent with previous determinations22. The latter case, i.e. for local couplings, is equivalent to the Edwards-Wilkinson growth model, however, the quenched nature of the noise induces a major change in the roughness exponent $\zeta$, from $\zeta=0.5$ for an annealed noise to 1.2 for a quenched noise.

Fisher et al.15 proposed a renormalization group analysis of this problem and obtained

$$
\zeta_{F}=\frac{2 \alpha-3 D}{3}
$$

where $D$ is the space dimension, i.e. in our case $D=1$. Expression (7) has been obtained by replacing the Laplacian propagator $1 / q^{2}$ by $1 / q^{\alpha-D}$ in the original calculation in order to take into account the long-range coupling. This theoretical prediction is plotted as a dotted line in Fig. 周. We observe a fairly good agreement for $1<\alpha<3$. However, we observe a marked difference for the Laplacian case, where the theoretical prediction $\zeta_{F}=1$ lies outside of the error-bars of our measurement.

The "roughness exponent" can be measured experimentally. In the case of wetting on heterogeneous surfaces in a Hele-Shaw geometry, A. Paterson et al.14 reported $\zeta=0.77$. Above a scale equal to the aperture of the cell, the problem should be described by the case $\alpha=3$. However, gravity plays a significant role in this problem introducing an additional confining term cutting down large scale front fluctuations. It is interesting to note that when gravity effects are suppressed (horizontal cell) the geometry of the invading fluid is similar to that of invasion percolation. The relation between invasion percolation and the quenched Edwards-Wilkinson problem has been discussed by Roux and Hansen22, in relation with a model of this process proposed by Čieplack 27. It may be that the expected scaling is restricted to a rather narrow window limited from below by the cell thickness, and from above by the capillary length (measuring the effect of gravity as compared to the surface tension). In case of wetting in a free surface geometry $(\alpha=2)$, E. Rolley et al.28 have found $\zeta \approx 1 / 3$, in very good agreement with our numerical result $(\zeta=0.35)$ Contrary to the explanation proposed by Robbins et al.29, using Imry and $\mathrm{Ma}$ arguments, our explanation takes into account the local dynamics of the chain, and may be extended by the same way to any range of the couplings. Another experimental situation which might be compared to our computation is the propagation of an interfacial crack. Such a situation between two PMMA plates has been studied recently by Schmittbuhl et alles. They measured $\zeta \approx 0.55$, a value which is much higher than the above mentioned value $\zeta=0.35$ for $\alpha=2$. The visco-elastic mechanical behavior of PMMA, or the initiation of cracks ahead of the front which merge with the front giving rise to a tortuous geometry where higher order terms may be relevant, may be responsible for this difference. Not also that Ref 26 is irrelevant in our case because the crack is not interfacial there.

\section{DYNAMICAL ORGANIZATION}

Starting with an uncorrelated distribution of forces, the system organizes after a transient into a correlated statistically stationary state. In particular, the memory of the initial state is lost and the distribution of forces $f(x)$ reaches a stationary distribution shown in Fig. (6). The forces have a critical value $F_{\max }$ depending on the value of $\alpha$. The distribution is asymetric. The higher the value of $\alpha$, the more peaked is the distribution close to $F_{\max }$. This distribution depends on the spatial correlations present along the front and on the distribution of force increment following an elementary move. The latter aspect can be taken into account 30 in a mean-field model, expected to hold for $\alpha<1$ as shown below. The maximum depinning force $f\left(x^{*}\right)$ as well (related to the external force by $\left.f\left(x^{*}\right)=-\eta_{i\left(x^{*}\right)}\left(x^{*}\right)-F_{\text {ext }}(t)\right)$ has a lower critical value corresponding to the maximal external force for the whole system, $F_{c}$. For a uniform constant depinning threshold for example $\left(\eta_{i\left(x^{*}\right)}\left(x^{*}\right) \equiv \eta_{c}=-1\right)$, the lower critical value of the maximum depinning force is $\left(1-F_{c} / L\right)$. This has been drawn in Fig. (6). $F_{c}$ increases with $\alpha$.

\section{PROPAGATION OF INSTABILITIES}

\section{A. Spatio-temporal map of activity}

As shown in Fig. (2), the active sites are spatially and temporally correlated. These correlations can be trivial as when $\alpha=0$, or more complex for $\alpha>1$. In order to analyze these correlations for various values of $\alpha$, it is of interest to study the probability distribution 31 of having a distance $r$ between the sites active at time $t$ and $t+\Delta t$ (i.e. the spatial distribution of the $\Delta t^{\text {th }}$ active site). From the numerical simulations, we observe that it is possible to describe the entire dependence of $p(r, \Delta t)$ for $\alpha>1$ through a scaling form

$$
p(r, \Delta t)=\Delta t^{-1 / z} \phi\left(\frac{r}{\Delta t^{1 / z}}\right)
$$

with a dynamic exponent $z$ which describes the spreading of the activity with time over a zone of size $\xi \propto \Delta t^{1 / z}$. For $\alpha \rightarrow \infty, z=2$, as for a diffusive system. Fig. (7) shows a data collapse for $\Delta t$ ranging from 1 to 64 and for $\alpha=2$. The exponent $z$ is determined from this collapse and it varies continuously with $\alpha$. The scaling function $\phi$ displays the following behavior:

$$
\phi(x) \propto \begin{cases}x^{-b} & \text { for } \quad x \gg 1 \\ x^{0} & \text { for } \quad x \ll 1\end{cases}
$$


For distances larger than the active length $\xi$, the decay of the scaling function is characterized by an exponent $b$ which also depends on $\alpha$. Fig. (8) summarizes the dependence of $b$ and $z$ on $\alpha$.

Let us first propose some arguments that allow us to understand the different observed regimes as a function of $\alpha$.

First, it is possible to link the dynamical roughness exponent $\zeta$ to the dynamical exponent $z$ in our model. Let us consider a starting point $\left(x_{o}, t_{o}\right)$. After a time $\Delta t$, the activity as spread over a distance $\xi(\Delta t)$ around $x_{o}$. The number of moves which have been necessary to cover the area between the crack fronts at time $t_{o}$ and $t_{o}+\Delta t$ scales as $\Delta x . \Delta u$. The front has a self-affine geometry with a roughness exponent $\zeta$, hence $\Delta u \propto \Delta x^{\zeta}$. As a result, we get the scaling of the time difference with the extent $\Delta x=\xi$ as

$$
\Delta t=\xi^{1+\zeta}
$$

hence

$$
z=1+\zeta
$$

This scaling relation is accurately obeyed in our numerical simulations as long as $0 \leq \zeta \leq 1$. For larger values of $\zeta$, the above scaling breaks down, and $\Delta u \propto \Delta x$, hence, the effective exponent $z=2$ appears.

Second, if we make the assumption that in the steady state there is only weak spatial distortions in the force distribution, then $p(r, \Delta t=1)$ should essentially reflect the load sharing rule due to the elastic coupling. This argument simply predicts

$$
b=\alpha
$$

as found in the numerical simulations for $\alpha<3$. An analogous relation has been found in the propagation of initially localized perturbation in the elastic map, without pinning 32 .

Third, as $\alpha$ increases, the load redistribution is much higher for the nearest neighbors than for the rest of the chain. More precisely, when $\alpha=3$ the interaction force

$$
f(x)=-k \int \frac{\left(u(x)-u\left(x^{\prime}\right)\right)}{\left|x-x^{\prime}\right|^{3}} d x^{\prime}
$$

contains a singular part proportional to

$$
f_{\text {sing }}(x) \propto\left(\frac{d^{2} u}{d x^{2}}\right)
$$

plus a regular part. The same holds for any higher value of $\alpha$, up to $\alpha$ tending to infinity where $f(x) \propto d^{2} u / d x^{2}$. Hence, the dynamics of the chain is essentially controlled by this singular part, and thus is no longer expected to depend on $\alpha$ but rather should be equivalent to the case $\alpha=3$ or $\alpha=\infty$, i.e. the simple Laplacian (local) kernel, or the Edwards-Wilkinson equation with quenched noise universality class. This regime will be referred to as Laplacian, or "L" regime, in the following.

Let us now return to distribution of distances between active sites at a time interval $\Delta t$. We observed the existence of a region of extent $\xi \propto \Delta t^{1 / z}$ centered on the initiation site where most of the activity is confined, we call this region "cluster" - although it is not connected in the space-time map. These clusters have a self-affine structure. Their scaling is indeed given by $\Delta x \propto \xi \propto \Delta t^{1 / z}$. We note that when $r>\xi$, the last occupied site is not part of the cluster which originates on the former site. The way the activity is distributed in space and time is controlled by the statistical distribution of the elementary jumps between two consecutive active sites which displays a very wide distribution, and the temporal correlations in those jumps. There are two limit cases which have been thoroughly explored, and which may serve as guides in the analysis: one case focuses on temporal correlations with a narrow (say Gaussian) distribution of elementary jumps, and the absence of a typical time scale would induce that the activity can be described as a selfaffine profile in time, with a roughness exponent $1 / z$. The other limit case corresponds to the absence of temporal correlations focusing on the power-law distribution of elementary jump, and where the space-time map of activity can be seen as a Levy walk. We do not know of any theoretical attempt to combine these two aspects to get a general picture. Due to the nature of the elementary jump distribution, it is natural to explore the second limit case as a reference, and check its domain of validity. Thus we now consider the crude hypothesis that temporal correlations can be neglected. The probability $p(r, \tau)$ can thus be written as the convolution of $p(r, 1)$ with itself $\tau$ times. For large $\tau$, the distribution of $p(r, \tau)$ will therefore converge to a statistical distribution which is stable for addition. If the distribution $p(r, 1)$ has a finite second moment, then $p(r, \tau)$ should converge to a Gaussian law as a result of the central limit theorem. However, this is never the case, since the above scaling implies that this would be obtained for $\alpha>3$, and we have seen that this case is similar to $\alpha=3$. In the intermediate case where $1 \leq \alpha \leq 3$, we have seen above that $p(r, \tau)$ decays as $r^{-b} \propto r^{-\alpha}$, and hence, the second moment of $r$ would diverge in an infinite system size. As a result, the central limit theorem does not apply, but rather $p(r, \tau)$ converges to a stable Levy law characterized by a power-law decay as $r^{-\alpha}$ for all $\tau$. Hence, the power-law tail will be preserved, for large distances, i.e. as long as the correlation length is smaller than the system size, $\xi<L$. This is indeed what is observed for $\alpha>1$. Still in the case where we neglect time correlations, we can relate the dynamic exponent $z$ to the large distance power-law decay through

$$
z=b-1=\alpha-1
$$

We see from Figure (8) that away from the value $\alpha=3$, where $z \approx 2$, the above relation is poorly satisfied. This 
indicates that temporal correlations becomes more and more important as $\alpha$ decreases, and cannot be neglected.

When $\alpha$ approaches 1 , all moments of the distribution become controlled by the system size, and hence after a few time steps, $p(r, \Delta t)$ is smeared out over the entire domain size and no more power-law tail survives. The scaling given by Eq.(8) indeed breaks down for $\alpha \leq 1$. As a natural consequence, time correlations also vanish, and we enter a Mean-Field (MF) regime, which is independent of $\alpha$.

From these arguments, we arrive at a classification of different regimes depending on the value of $\alpha$ :

- $\alpha \geq 3$ the Laplacian regime, or the Edwards-Wilkinson regime with a quenched noise, where the kernel is equivalent to a second derivative.

- $1<\alpha<3$ an intermediate regime, where the critical exponents which are measured depend continuously on $\alpha$.

- $\alpha \leq 1$ the Mean-Field regime, where spatial correlations are lost in a few time steps, and where - as in the L-regime - the value of $\alpha$ does not influence the critical exponents.

\section{B. Activity recurrence}

In the critical steady state, the activity map is highly correlated and exhibits scale invariant features in both time and space. We studied previously the spatial distribution of activity after a fixed time lag $\Delta t$. We now turn to a complementary description, focusing on a single site as a function of time. More precisely, if site $i$ was active at time $t_{o}$, we study the distribution, $p_{F I R S T}(t)$, of the time delay $t$, such that the next move at site $i$ occurs at time $t_{o}+t$. Fig. (9) shows such a distribution for $\alpha=1,1.5,2,3$ and $\infty$. The very early time behavior is dependent on the distribution of displacement $u$ which is chosen. If the displacement is distributed over the interval $[a, b]$, for large $a$, we prevent the recurrence of activity immediately after a move. However, this effect lasts only for a time much lower than the maximum time $T^{*}$ when $\alpha>1$. It does not affect the distribution over a large interval depending on the system size, and thus can be discarded from the analysis. Then for $\alpha>1$, we observe a power-law decay

$$
p_{F I R S T}(t) \propto t^{-\tau_{F I R S T}}
$$

where $\tau_{F I R S T}<2$. This power-law terminates at an upper cut-off which scales as the time necessary for a cluster to span the entire system size, $T^{*}=L^{z}$. Let us now summarize our observations for various $\alpha$ values.

- $\alpha>3$ Consistently with the previous discussion, the value of the exponent $\tau_{F I R S T}$ is independent of $\alpha$ and amounts to $\tau_{F I R S T} \approx 1.5$ in good agreement with previous studies.

- $2<\alpha<3$ The distribution drops rapidly (faster than any power-law) above the cut-off scale $T^{*}$. The exponent
$\tau_{F I R S T}$ progressively increases and reaches the value 1 as $\alpha$ tends to 2 .

- $1<\alpha<2$ The exponent $\tau_{F I R S T}$ saturates to 1 , for times up to $T^{*}$, but in contrast to the previous cases, the distribution then reaches a plateau from $T^{*}$ to $T^{* *}$, before dropping faster than any power-law. The scaling of this second time scale (Fig. 10) is identical to that of $T^{*}, T^{* *} \propto L^{z}$. The relative importance of the $1 / t$ region and of the plateau, can be estimated by the ratio $T^{*} / T^{* *}$ which goes from 1 (no plateau) to 0 (no power-law) as $\alpha$ decreases from 2 to 1 .

- $\alpha<1$ We enter the Mean-Field regime where $p_{F I R S T}$ is constant for times up to $T^{* *}$ which is proportional to the system size $L$. Again this is consistent with the meanfield regime previously discussed.

It is interesting to note that the intermediate regime is now split in two cases, with a short and long time behavior differing for $1<\alpha<2$ rather than a continuous evolution with an exponent $\tau_{F I R S T}$ going from 1 to 0 . The flat plateau regime observed for $T^{*}<t<T^{* *}$ and $1<\alpha<2$ is similar to the Mean-Field regime for $\alpha<1$, and thus the transition to the MF regime appears not to be as brutal at $\alpha=1$ as proposed from the scaling of the distance, but rather it turns out to be gradual, with a mixed regime displaying non-trivial correlation for a "macroscopic" time $T^{*}$, before reaching a MeanField behavior at later times. The surprising feature is that space and time correlations do not disappear simultaneously. It might be interesting to note that the mean distance between active sites separated by a delay $\Delta t$ also changes for $\alpha$ smaller or larger than 2. For $\alpha>2$, it follows from the previous spatio-temporal analysis (part A), that the mean distance between active sites scales as the correlation length $\xi$ since $b>2$. On the contrary, for $\alpha<2$, it scales as the system size $L$.

Let us consider an active site $i$ at time $t_{0}$. After a time $t$, the number of times, $n(t)$, the site $i$ has been active can be estimated as the total number of individual step proportional to $t$ divided by the number of sites where most of the activity is concentrated, i.e. $t^{1 / z}$, or

$$
n(t) \propto t^{1-1 / z}
$$

Let us now estimate the same number using the distribution of activity recurrence $T$. From the power-law distribution of $T$, we can estimate the maximum among $n(t)$ numbers - supposed to be uncorrelated - from

$$
\int_{T_{\max }(n)}^{\infty} p_{F I R S T}(T) d T \propto \frac{1}{n(t)}
$$

or $T_{\max }(n) \propto n^{1 /\left(\tau_{F I R S T}-1\right)}$. Using the fact that $\tau_{F I R S T}$ is always smaller than 2 , we can compute the mean time between activity recurrence $\langle T\rangle \propto T_{\max }^{2-\tau_{F I R T}} \propto$ $n^{\left(2-\tau_{F I R S T}\right) /\left(\tau_{F I R S T}-1\right)}$. This same average time is also equal to $\langle T\rangle=t / n(t)$. Equating these two estimates gives

$$
n(t) \propto t^{\tau_{F I R S T}-1}
$$


Comparing Eqs. (16) and (18), we arrive at the following expression for $\tau_{F I R S T}$ :

$$
\tau_{F I R S T}=\frac{2 z-1}{z}
$$

This estimate is based on the fragile assumption that time correlations can be neglected. We have seen above that a similar hypothesis lead to $z=\alpha-1$, from what we deduce

$$
\tau_{F I R S T}=\frac{2 \alpha-3}{\alpha-1}
$$

This expression however requires that the distribution of $T$ is not governed by the upper cut-off $L^{z}$, and thus $\tau_{\text {FIRST }}>1$ strictly, or $\alpha>2$ in the above formula. Figure (11) shows the measured value of $\tau_{F I R S T}$ as a function of $\alpha$, together with the above relation. We observe a reasonnable agreement for $2<\alpha<3$.

\section{Avalanche Dynamic}

Up to now, we have considered an ideal driving where the front displacement is controlled by adjusting instantaneously the driving force. Assuming an overdamped dynamics, we can reconstruct a different type of driving.

In order to proceed, it is useful to introduce the notion of avalanches or bursts. Let us consider the sequence of external force $F_{\text {ext }}(t)$. Any force $F$ will split the signal into consecutive intervals with alternating $F<F_{\text {ext }}$ domains (or "obstacles"), and $F>F_{\text {ext }}$ intervals (or "bursts"). Each burst is thus the front motion which would result from a constant force $F$ being imposed to the system.

Avalanches were introduced initially 33 in order to understand some features of invasion percolation 31 . However, in Ref 33 , some approximations were unjustified or unfounded. A complete solution of the avalanche statistics applied to invasion percolation and other extremal models is presented in Paczuski et a 23. The key property is that it is possible to relate the avalanches in the external forcing to the cluster statistics in some extremal models such as invasion percolation. The avalanche size distribution at fixed $F$ is a power-law distribution up to a maximum avalanche size which diverges as $F$ approaches to a critical value $F_{c}$. This mapping allows to derive geometrical information on the activity from a simply accessible external signal, the driving force. One amazing feature is that the latter is one-dimensional but encode a multi-dimensional information.

In our case, it is unfortunately impossible to establish such a direct mapping because of the non-local nature of the interaction. Hence, the connectedness of the clusters is lost, or equivalently, the clusters to be defined from the driving force avalanches do not have a straightforward geometrical interpretation. Nevertheless, the maximum external force $F_{c}$ encountered in the external driving can still be identified with a critical point where the correlation length diverges. This critical point is a depinning transition. For a constant driving $F$ strictly smaller than $F_{c}$, the front will advance only over a finite distance and stop when it encounters a pinning force larger than $F$. Similarly, if $F>F_{c}$, the front will never be stopped.

It is also possible to use the time evolution of the system to define two distributions of avalanche which do not require the precise identification of the critical threshold. At each time step, $t$, one can construct the avalanches which correspond precisely to the loading $F(t)$. In fact, two such avalanches can be considered, for times either larger or slower than $t$, the forward and backward avalanches respectively as shown in Figure 12. Figure 14 shows both cumulative avalanche distributions for our model with $\alpha>1$. They behave as power laws

$$
N_{f, b}(t) \propto t^{1-\tau_{f, b}}
$$

with no upper cut-off apart from the system size.

Maslov 34 showed that for a variety of models, the exponent $\tau_{f}$ was super-universal, and equal to 2 . Consequently, forward avalanches do not reveal much information on the model. Hpyever, in the extremal models studied in Paczuski et al23, the backward avalanche exponent is not super-universal and it does give rise to a non trivial critical exponent $\tau_{f}$.

In our numerical results, the universal result $\tau_{f}=2$ is observed for $\alpha>1$. The backward value is also measured to be $\tau_{b}=2$ for the same values of $\alpha$.

Such power-laws with exponents $\tau_{f}=\tau_{b}=2$ also appear in a series of uncorrelated random numbers picked from the same distribution, however, we cannot infer from this result that the forces are uncorrelated. They are indeed strongly correlated as can be seen from the power spectrum of the signal $F_{\text {ext }}(t)$ (Fig.15) which shows a power-law extending over long time intervals (yet smaller than the time needed to span the system $L^{z}$ ). If we were to interpret this signal as self-affine we would estimate from such a graph a self-affine Hurst exponent $\zeta_{F}=-0.4$, a negative exponent which means that the signal is strongly anti-correlated. The same power-law appears for all values of $\alpha$ larger than 1 . One has to be cautious while manipulating Hurst exponent which are negative, since most properties relying on the selfaffine nature of a signal break down in this case. In fact for most properties, such functions behave as if the Hurst exponent was actually zero (white noise). (Otherwise, in order to reach reliable conclusions, one may consider the time-integrated force signal i.e. the energy dissipated during the front motion. More precisely, the mean force can be substracted from $F$ before integration so that only the fluctuating part of the energy is considered. For $\alpha>1$, this signal has a self-affine exponent $\zeta_{F}+1 \approx 0.6$, which can be measured using standard tools.) The avalanches are thus expected to behave as if the force signal was a white noise, hence $\tau_{f}=\tau_{b}=2$. 
For small exponent $\alpha<1$, there is a short time (high frequency) behavior which seems to follow a similar powerlaw behavior. The plateau appearing for very small times is probably due only to numerical accuracy and has not to be considered. But at large times, the power spectrum of the force crosses over to a $\omega^{-2}$ regime which is the signature of a $\zeta_{F}=0.5$ self-affine signal, analogous to a random walk. The avalanche exponents are consequently changed to $\tau_{f, b}=1.5 \pm 0.05$ for $\alpha<1$.

Although the previous analysis of avalanches can in principle be accessed experimentally, it requires to be able to switch from a displacement-controlled to a forcecontrolled mode, what is in general a rather difficult requirement. There is another, more natural, way to have access to the fluctuating driving force through the intermittent front motion. We have seen that a constant force control for finite size system either lead to a stop or a constant motion, the transition between the two regime corresponding to the maximum $F_{\text {ext }}$. Thus a single configuration will determine the threshold, and for a finite size system, this single point generally is not representative of the critical behavior, and it contains a rather poor information. A way to circumvent this difficulty is to introduce a small but non-zero stiffness $\epsilon$ in the control (Fig.13), so that as the front advances, the driving force is progressively decreased by a quantity $\epsilon .\langle u\rangle$. This insures that the motion will never be unlimited. As soon as the front is pinned, then the driving force is slowly increased up to the depinning limit. If $\epsilon$ is small enough, only the forces close to the pinning threshold will be probed. In addition, the statistics of the front advance between two pinned configuration gives some information on either the correlations of $F_{\text {ext }}$, or on its statistical distribution close to the critical threshold.

We performed such an analysis for various values of $\alpha$. We observed that the avalanches have a maximum size $s^{*}$ which depends on $\epsilon$ as

$$
s^{*} \propto \epsilon^{-\kappa}
$$

with

$$
\kappa \approx 0.65
$$

for all values of $\alpha$ investigated from 1 to 4 . This maximum size can be used to scale the avalanche size $s$ (Fig.15), and thus it allows to account for the distribution in an $\epsilon$ independent manner

$$
n_{\epsilon}(s) \propto \frac{1}{s^{* r}} \psi\left(\frac{s}{s^{*}}\right)
$$

where $\psi$ is a power-law distribution for small arguments $\psi(x) \propto x^{-\theta}$ for $x \ll 1$, and decays rapidly to zero for $x \gg 1$. And $s^{* r}$ is a normalization factor.

There are different features which may contribute to the statistics of $s$. We here focus on one aspect of the problem namely the statistical distribution of the pinning force close to the depinning critical point. Note that the results which could be derived from the self-affine structure of the signal $F_{\text {ext }}(t)$ are not valid due to the asymmetry of the distribution of forces, $p\left(F_{\text {ext }}\right)$ (Fig.6).

Let us note

$$
p\left(F_{\text {ext }}\right)=\left(F_{c}-F_{\text {ext }}\right)^{\beta} \equiv \delta^{\beta}
$$

the distribution of external forces near the threshold $F_{c}$. After $n$ events, the system has lost an elastic force $\delta$,

$$
\delta=n \epsilon
$$

The maximum force encountered follows

$$
\delta^{-(1+\beta)}=n
$$

Balancing the two expressions leads to

$$
\epsilon=n^{-1 /(1+\beta)-1}=n^{-(2+\beta) /(1+\beta)}
$$

hence

$$
\kappa=\frac{1+\beta}{2+\beta}
$$

From numerical data, we have $\beta \approx 1$. It leads to

$$
\kappa \approx 0.66
$$

in good agreement with Eq. (23), although temporal correlations have not been considered here.

\section{DISCUSSION}

We have presented in this article a study on the correlations induced by the dampened motion of a finite size elastic chain with long range interactions and driven along its transversal direction on a surface with quenched disorder.

We have shown that the system organizes after a transient in a stationary state with long-range correlations. The memory of the initial state is lost. The final state does not depend on the initial state, but its characteristics depend on $\alpha$. We have pointed out three regimes: For very long range couplings $(\alpha \leq D)$, a regime controlled by finite size effects and analogous to a Mean-Field one. For very short range couplings $(\alpha \geq D+2)$, a regime controlled by small distance singularities of the Green function and displaying the same characteristics as Laplacian couplings. In between, $(D<\alpha<D+2)$, an intermediate regime where characteristic exponents $b, z$ and $\zeta$ evolve continuously with $\alpha$. The transition between the three regimes of the activity map is a new one. It is summarized in figure 17. The transition to a Mean Field uniform regime does not appear simply for $\alpha=3 D / 2$ as been proposed by D.Fisher et al.3 by Functional Renormalization Group analysis, but it appears clearly from the simulations, that spatial and temporal correlations do not disappear simultaneously. The transition to the 
Mean Field regime is characterized by a cross-over timelength $t_{c}$ growing with $\alpha$, above which the uniform distribution is valid and below which the power law behavior with non trivial exponent is observed. Even if the strong pinning assumption ensures that the instabilities are local, when $t_{c}$ reaches small times, the behavior of the system becomes "delocalized". The "delocalized" behavior refers to a uniform spreading of activity over the system above $t_{c}$. For $\alpha>2$, the system exhibits "collective" organization (characterized by long-range correlations) but "localized" behavior. For $\alpha<1$ the system exhibits a uniform "delocalized" behavior analogous to the Mean Field one. For $1<\alpha<2$ the system exhibits collective organization with distortions at small scales but "delocalized" behavior at large scales (above $t_{c}$ ). Moreover, the time $t_{c}$ goes to infinity in the thermodynamic limit: it scales as the activity spreading time $L^{z}$ and does not introduce a new scaling. Its origin remains unclear to us. An analogous transition appears in the external force for $\alpha<1$ (see Figure15).

Modifying the parameters of the system, combination of the above behavior can also be found. For a mix of various couplings for example, the system exhibits a crossover between a regime controlled by the short range coupling (at small time and small length) and a regime controlled by the long range coupling (at large time and large length). The transition between these two regimes is size independent and the spatial and the temporal transition are related by the dynamical exponent of the shortest couplings.

The results presented above have been obtained with a discretized system and periodic boundary conditions. We have checked that the same results are obtained, far away of the boundaries, without periodic boundary conditions. Particularly, the exponents obtained are the same in this case. The temporal discretization and the breakdown of the translational invariance (because only one site is allowed to jump at each time step) are not limiting. They are consistent with the "strong pinning assumption". At this stage, the simulations do not allow us to justify this assumption more strongly. We know however 30 that there exists a transition from weak to strong pinning which could be used to justify a modeling at larger scales. But for very long range (small $\alpha$ ) and small system size we may encounter situation of weak pinning, a case out of reach for our model. We suppose that the local curvature of the pinning potential is always sufficiently high to maintain the system in the strong pinning limit. In this limit, the results presented here appear to be universal, provided some quenched disorder is introduced: the choice made for the distribution $u$ and for the time step only affects short distances. As has been mentioned, they are important in the mean field regime, in agreement with ref.B but they do not affect the position of the transition to the Mean Field behavior. When no disorder is introduced, the system evolves toward a limit cycle of period $L$. The boundary conditions affect the distributions only at the proximity of the upper cut-off scale, $L$ or $L^{z}$, and the displacement fields near the borders. When disorder is present, it is possible from our numerical results to find intermediate scales where the behavior of the system is characterized in space and in time by power law distributions. The critical exponents of the distributions only depend on $\alpha$ as discussed above. More surprisingly, due to the temporal anticorrelation in the threshold force, the internal avalanche distribution seems to be independent of $\alpha$ and does not reflect the correlations of the external force. A better accuracy is restored with the help of an elastic external driving. This gives rise to external avalanches. Let us mention that velocity has to be defined carefully in the case of driving with constant external force 35 .

Let us turn to the notion of multistability. For a one particle system, the multistability is due to the non linear concurrent effect of the elastic force and of the pinning force. The condition of multistability can be given by a linear stability analysis. The particle is unstable when the negative curvature of the pinning potential ovefcomes the elastic stiffness. It leads to hysteretic behaviorl. The energy dissipated at very low velocity is related to the area of the hysteretic cycle. For a $N$-particle system, the instability involves more than one particle, due to the coupling between asperities. The size of the domain involved in the instability depends on the distortions in the curvature of the pinning potential and on the range of the elastic interactions. In the "weak pinning limit", the one-particle instability condition is not effective, but multistability can occur at larger scale. The minimal size giving rise to multistability is usually computed by the "Larkin Method" t. This length is associated to a dynamic by blocks whose existence remains controversial in the literature. In the "strong pinning limit", the one particle case is effective, but the elastic coupling may propagate the instability to an avalanche of larger size. We showed here that the system thus organizes into a "critical state". There is no intrinsic length scale in such states, but the lowest cut-off and the system size. This effect gives rise to "kinetic roughening" in finite systems and has been well studied in the case of short range elastic interactions for various type of pinning 16 . It does not induce block motion involving more than one asperity. An interesting question would be: is the "block dynamics" of the weak pinning limit analogous to the strong pinning dynamics? This question remains open.

\section{CONCLUSION}

We have shown in this paper that in the strong pinning limit and for a quasi-static driving, a finite elastic system driven on a disordered surface exhibits long range dynamical correlations. This can be shown by the roughening of the surface, in agreement with spreading or crack front propagation experiments. It can be studied numerically by the statistical analysis of the Activity 
Map. The range $\alpha$ of the interaction allows to interpolate between a Laplacian dynamical regime (for $\alpha>\alpha_{c 2}$ ) and a Mean Field dynamical regime (for $\alpha<\alpha_{c 1}$ ). In our one-dimensional simulations, with quenched decorrelated disorder, $\alpha_{c 1}=1(=D)$ and $\alpha_{c 2}=3(=D+2)$. The transition to the Mean Field regime appears to take place progressively from the large times when $\alpha<2$. Space and time correlations do not disappear simultaneously.

The internal avalanche size distribution seems to be "superuniversal" (independent on $\alpha$ ) because it does not preserve informations on the temporal correlations in the threshold force and exhibits the simple $1 / f^{2}$ behavior characteristic of jumps. The dependence on $\alpha$ is recovered by an elastic external loading.

This study shows one example of physical systems where microscopic disorder leads to large scale structures as can be revealed by kinetic roughening (large scale displacement fluctuations) or avalanche distributions. The algebraic decay of the interactions determines the critical exponents but other details are unimportant in the scaling behavior. The large scale power-law behavior is a signature of the self-organization of the system.

This study was designed to describe a "strong pinning" limit. It would be of interest to extend the model to the weak pinning case.

\section{ACKNOWLEDGMENTS}

It is a pleasure to acknowledge fruitful discussions with J. Schmittbuhl, B. Protas, S. Krishnamurthy, H.J. Herrmann and M. Fermigier. This work is partly supported by the Groupement de Recherche "Physique des Milieux Hétérogènes Complexes" of the CNRS.

${ }^{1}$ A. I. Larkin and Yu. N. Ovchinnikov, J. Low Temp. Phys., 34, 409 (1979). For a review, see T. Giamarchi and P. LeDoussal in Spin Glasses and Random fields edited by A. P. Young, World Sci. ( Singapore,1997).

${ }^{2}$ S. Zapperi, P. Cizeau, G. Durin and H. E. Stanley, condmat/9803253.

${ }^{3}$ D. S. Fisher, Phys. Rev. B, 31, 1396 (1985).

${ }^{4}$ J. P. Bouchaud, E. Bouchaud, G. Lapasset and J. Planes, Phys. Rev. Lett., 71, 2240 (1993).

${ }^{5} \mathrm{C}$. Caroli and $\mathrm{Ph}$. Nozieres in The physics of sliding friction edited by B. N. J. Persson, Vol.311 of NATO Advanced Study (Kluwer, Dordrecht,1996).

${ }^{6}$ P. Mazur and I. Oppenheim, Physica, 50,241 (1970).

${ }^{7}$ J. F. Joanny and P. G. de Gennes, J. Chem. Phys., 81, 552 (1984).

List of Captions:
${ }^{8}$ J. Schmittbuhl, S. Roux, J.-P. Vilotte and K.J. Måløy, Phys. Rev. Lett., 74, 1787 (1995).

${ }^{9}$ H.Gao and J.R.Rice, J. of Appl. Mech., 56, 828 (1989).

${ }^{10}$ L. Landau and E. Lifchitz, Théorie de l'élasticité, Mir Ed. (1990).

${ }^{11}$ P. A. Lee and H. Fukuyama, Phys. Rev. B, 17, 542 (1978).

12 C. Castellani, C. Di Castro and A. Maccarone, Phys. Rev. B, 55, 2676 (1997).

${ }^{13}$ O. Zik, E. Moes, Z. Olami and I. Webman, Europhys. Lett., 38, 509 (1997).

14 A. Paterson, Annales de Physique, 21, 337 (1996).

15 T. Nattermann, S. Stepanow, L. H. Tang and H. Leschhorn, J. Phys. II (France), 2, 1483 (1992); H. Leschhorn, T. Nattermann, S. Stepanow, L. H. Tang cond-mat/9603114; O. Narayan and D. S. Fisher, Phys. Rev. B, 48, 7030 (1993).

16 T. Nattermann and L. H. Tang, Phys. Rev. A, 45, 7156 (1992); E. Medina, T. Hwa, M. Kardar and Y. C. Zhang, Phys. Rev. A, 39, 3053 (1989); K. Sneppen, Phys. Rev. Lett., 69, 3539 (1992); M. Kardar, G. Parisi, Y. C. Zhang, Phys. Rev. Lett., 56, 889 (1986). For a review, see T. Halpin-Healy and Y. C. Zhang, Physics Reports, 254, 215 (1995).

17 A. Tanguy and S. Roux, Phys. Rev. E, 55, 2166, (1997).

${ }^{18}$ F. Heslot, T. Baumberger, B. Perrin, B. Caroli and C. Caroli, Phys. Rev. E, 49, 4973 (1994).

${ }^{19}$ D. Wilkinson and J. F. Willemsen, J. Phys. A, 16, 3365 (1983).

${ }^{20}$ H. J. Herrmann and S. Roux in Statistical models for the fracture of disordered media, Random Materials and Processes, Series Editors H. E. Stanley and E. Guyon (Elsevier Science Publisher, North-Holland, 1990).

${ }^{21}$ P. Bak and K. Sneppen, Phys. Rev. Lett., 71, 4083 (1993).

${ }^{22}$ S. Roux and A. Hansen, J. Phys I France, 4, 515 (1994).

${ }^{23}$ M. Paczuski, S. Maslov and P. Bak, Phys. Rev. E, 53, 414 (1995).

${ }^{24}$ H. Flyvbjerg, K. Sneppen and P. Bak, Phys. Rev. Lett., 24, 4087 (1993).

${ }^{25}$ J. Schmittbuhl and K. J. Måløy, Phys. Rev. Lett., 78, 3888 (1997).

${ }^{26}$ P. Daguier, B. Nghiem, E. Bouchaud and F. Creuzet, Phys. Rev. Lett., 78, 1062 (1997).

${ }^{27}$ M. Čieplack and M. O. Robbins, Phys. Rev. Lett., 60, 2042 (1988). M. Čieplack and M. O. Robbins, Phys. Rev. B, 41, 11508 (1990).

${ }^{28}$ E. Rolley, C. Guthmann, R. Gombrowicz and V. Repain, preprint (1997).

${ }^{29}$ M.O. Robbins and J.F. Joanny, Europhysics Lett., 3, 729 (1987).

${ }^{30}$ A. Tanguy, PhD Thesis, Université Paris VII (1998).

${ }^{31}$ L. Furuberg, J. Feder, A. Aharony and T. Jossang, Phys. Rev. Lett., 61, 2117 (1998).

32 A. Torcini and S. Lepri, Phys. Rev. E, 55, R3805 (1997).

${ }^{33}$ S. Roux and E. Guyon, J. Phys. A, 22, 3693 (1989).

${ }^{34}$ S. Maslov, Phys. Rev. Lett., 74, 562 (1995).

${ }^{35}$ E. Raphaël and P. G. de Gennes, J. Chem. Phys., 90, 7577 (1989). 
FIG. 1. (a) Schematic motion of the elastic chain in the force field $\eta(x, u(x))$ between $t$ (black) and $t+\Delta t$ (grey). The redistribution of the elastic forces via the long-range interactions is not visualized in this picture. (b) Schematic description of the pinning force for one site $x$. The abscisse is now the average position of the line.

FIG. 2. Activity map showing the location of the active sites as a funtion of time for various couplings $\alpha=1(\mathrm{a}), \alpha=2(\mathrm{~b})$ and $\alpha=3(\mathrm{c})$. The system size is $\mathrm{L}=1024$.

FIG. 3. Displacement front at different time intervals and for various couplings $\alpha=1(\mathrm{a}), \alpha=2(\mathrm{~b})$ and $\alpha=3(\mathrm{c})$. The system size is $\mathrm{L}=1024$.

FIG. 4. Average power spectrum of the displacement front position in log-log scale for a $\alpha=2$ coupling. The dotted line shows a power-law fit which corresponds to the roughness exponent $\zeta=0.35$. The system size is $L=1024$.

FIG. 5. Roughness exponent $\zeta$ versus coupling range $\alpha$, measured from the power law fits of the displacement correlation function.

FIG. 6. (a) Statistical distribution $n(f)$ of forces along the chain in the stationary regime, for various couplings $\alpha$. The scale for forces is given by the maximum of forces $\left|G\left(x^{*}, x^{*}\right) \cdot \max (u)\right|$. The pinning threshold is taken arbitrarily as $\eta_{i\left(x^{*}\right)}\left(x^{*}\right) \equiv-1$. (b) Contribution of the maximum depinning forces $f\left(x^{*}\right)=1-F_{\text {ext }}(t)$ (little $\diamond$ ) in the distribution $n(f)$ for $\alpha=1$. The dashed line stands for a fit of $n\left(f\left(x^{*}\right)\right)$ near the threshold $f\left(x^{*}\right)_{c}=1-F_{c} / L$ with $\beta=1$.

FIG. 7. Data collapse of seven different probability distributions $p(r, \Delta t)$ for $\alpha=2$ and time intervals ranging from 1 to 64 . The system size is $\mathrm{L}=512$. The redistribution exponent is $b=1.95$, and the best data collapse is obtained for $z=1.30$.

FIG. 8. (a) Plot of the $b$ exponent. (b) Plot of the $z$ exponent obtained for different values of $\alpha$. The dotted and dashed lines are the proposed asymptotic behavior discussed in the text. The dotted line $(2 \alpha / 3)$ on the right take account for the relation $z=1+\zeta$ and the expression proposed for $\zeta$ by Fisher et al.

FIG. 9. Log-Log plot of the first return probability distribution $p_{F I R S T}(t)$ as a function of the time $t$ for various coupling range $\alpha=1, \alpha=1.5, \alpha=2, \alpha=3$ and laplacian. The system size is $L=512$.

FIG. 10. Data collapse of the first return probability distribution for various system sizes for $\alpha=1.5$. The upper cut-off scale as $L^{z}$ as well as the extent of the plateau.

FIG. 11. Plot of the $\tau_{F I R S T}$ exponent obtained for different values of $\alpha$. Two different exponents appear for $1<\alpha<2$ in the Mixed Regime. They correspond to the distribution below and above $t_{c}$. The dotted lines are the proposed asymptotic behavior discussed in the text.

FIG. 12. External loading $F_{\text {ext }}(t)$ as a function of time. From this signal, on can define avalanches either by prescribing a fixed value of $f$, and considering the intervals where $f$ remains below this limit, or by constructing these avalanches starting from all forcing values $F_{\text {ext }}(t)$ with the statistics issued from the model, and choosing either a forward or backward time direction.

FIG. 13. Instantaneous loading $F_{\text {ext }}(t)$ as a function of time and external elastic load of stiffness $\epsilon$.

FIG. 14. Log-log plot of the distribution of the forward and backward avalanches for $\alpha>1$. The straight lines are power-law fits of exponents -2 . 
FIG. 15. Spectral amplitude of the time fluctuation of the time integration of the threshold force $F_{\text {ext }}(t)$ for various couplings $\alpha$ ranging from 0 to 3 . The straight line represents a power-law of exponent -2.2 which implies a roughness exponent of the direct time force signal of -0.4 for $\alpha>1$. The full line represents a power-law of exponent -4 which implies a roughness exponent +0.5 .

FIG. 16. Log-log plot of the distribution of external avalanches for $\alpha=2$ and for ten different stiffnesses $\epsilon$ ranging from $10^{-4}$ to $10^{-3}$. The straight line is a power-law fit of exponent -1.09 . The upper cut-off scales like $s^{*} \propto \epsilon^{-0.65}$.

FIG. 17. Schematic transition between the three apparent dynamical regimes. Dotted line separates the classical power law regime and a new one regime with a "local" behavior at short time and a "delocalized" behavior at large time. The Mixed Regime stands for a regime with Mean-field type character only for time correlations and at large times.
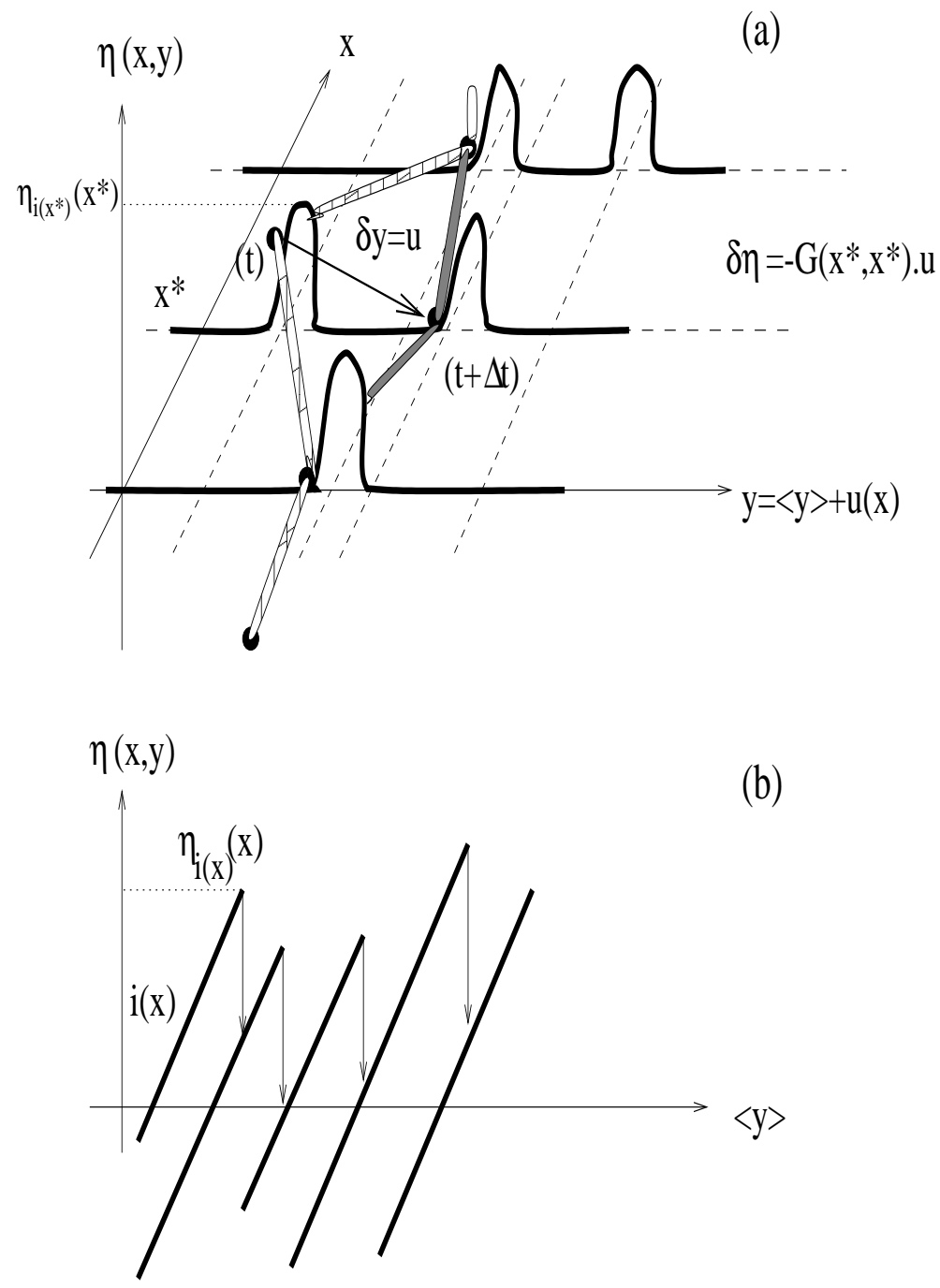

Figure 1 

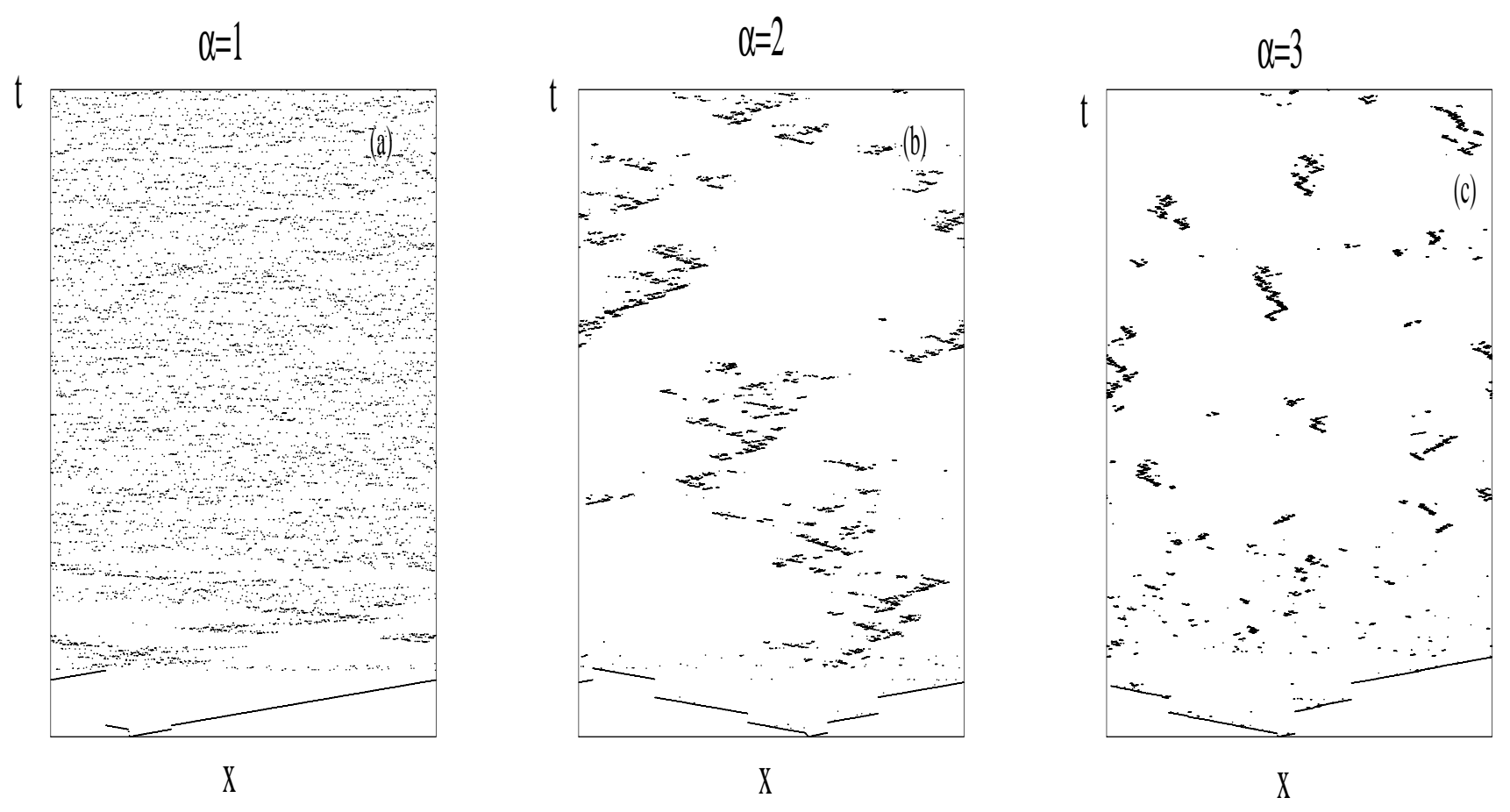

Figure 2
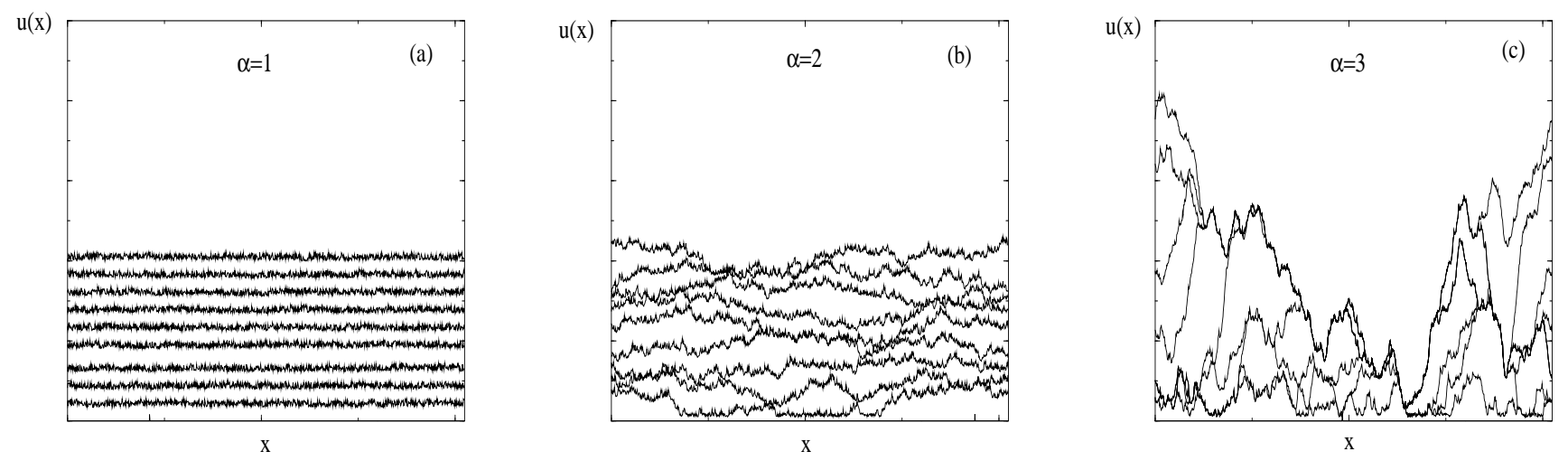

Figure 3 


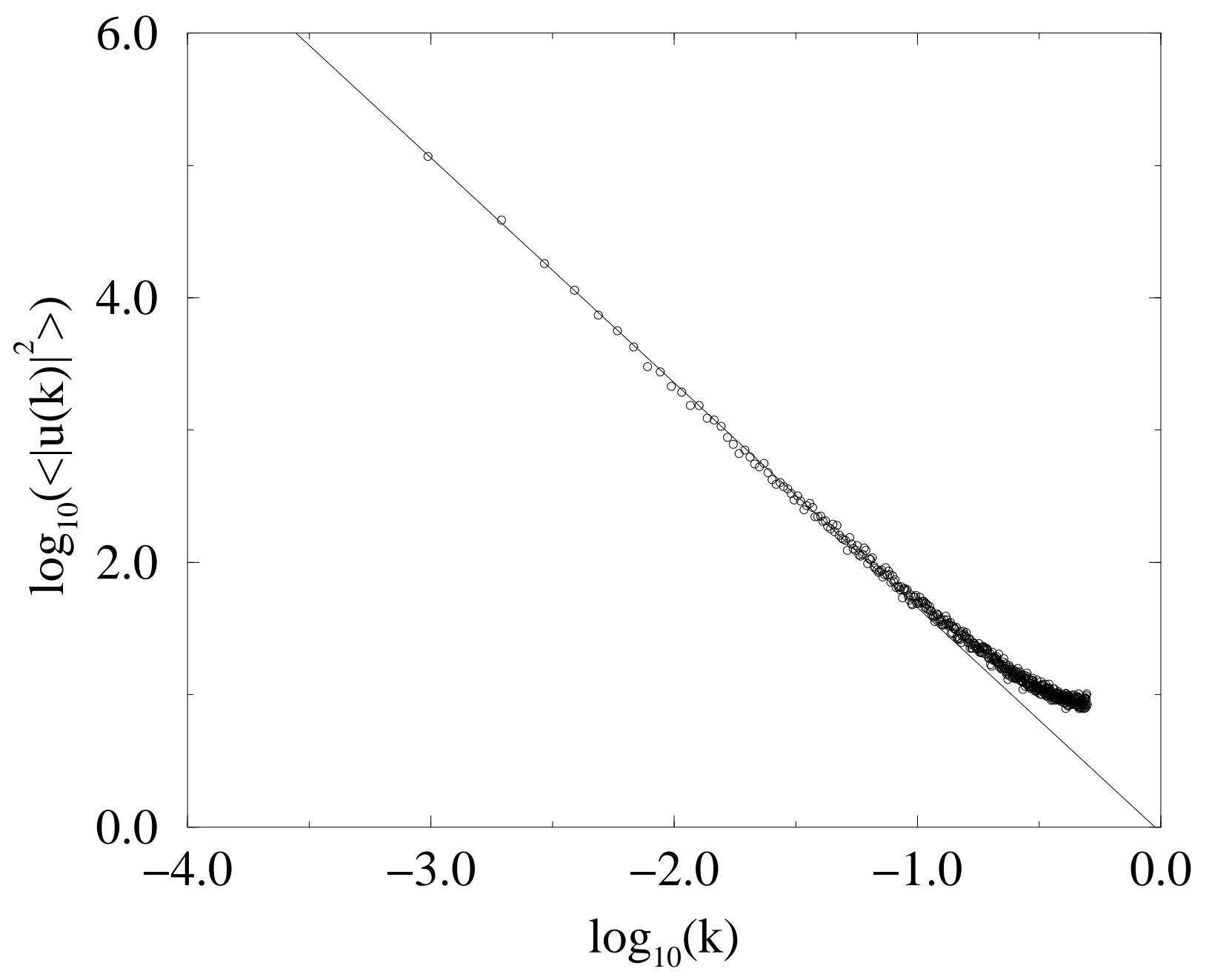

Figure 4 


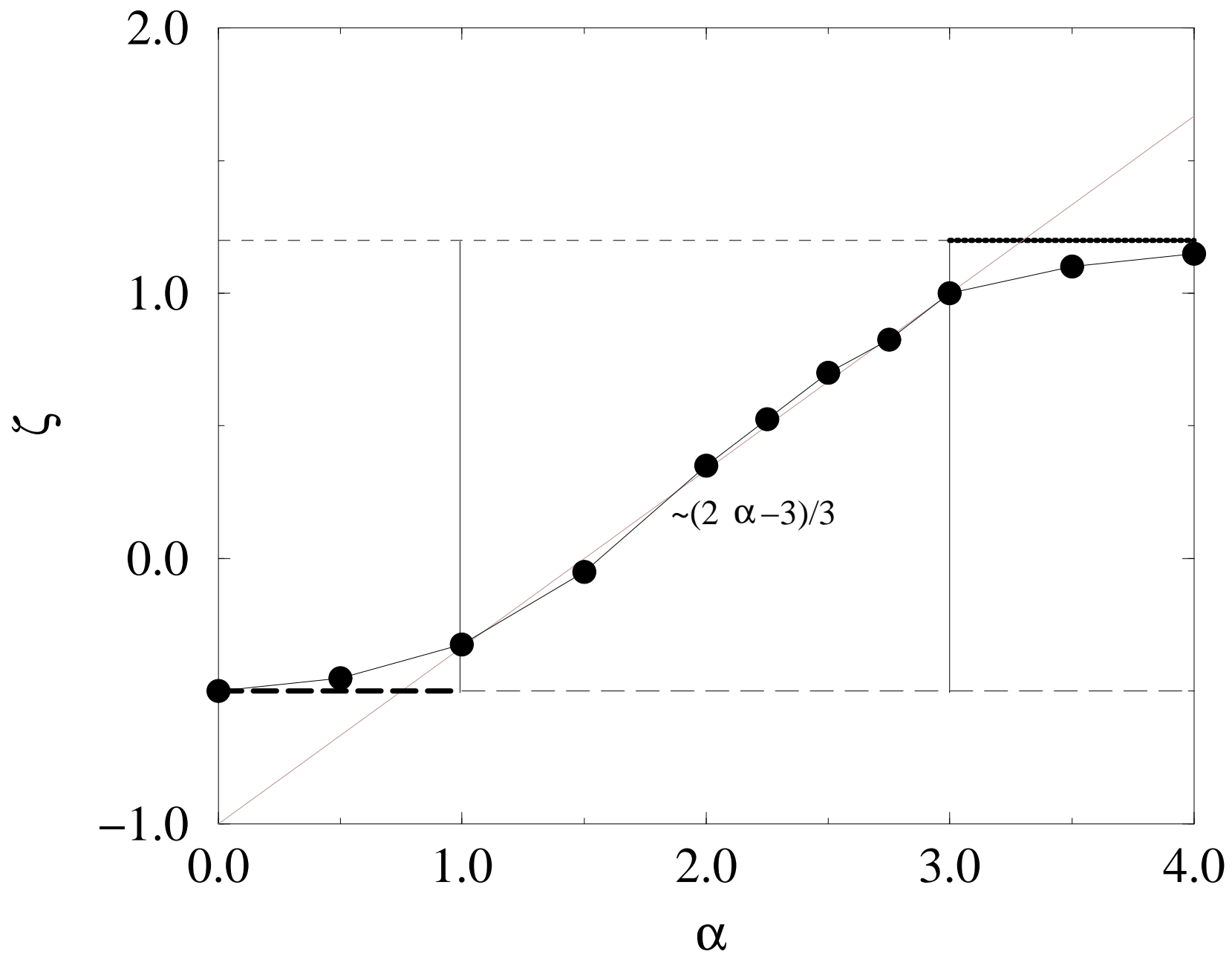

Figure 5 

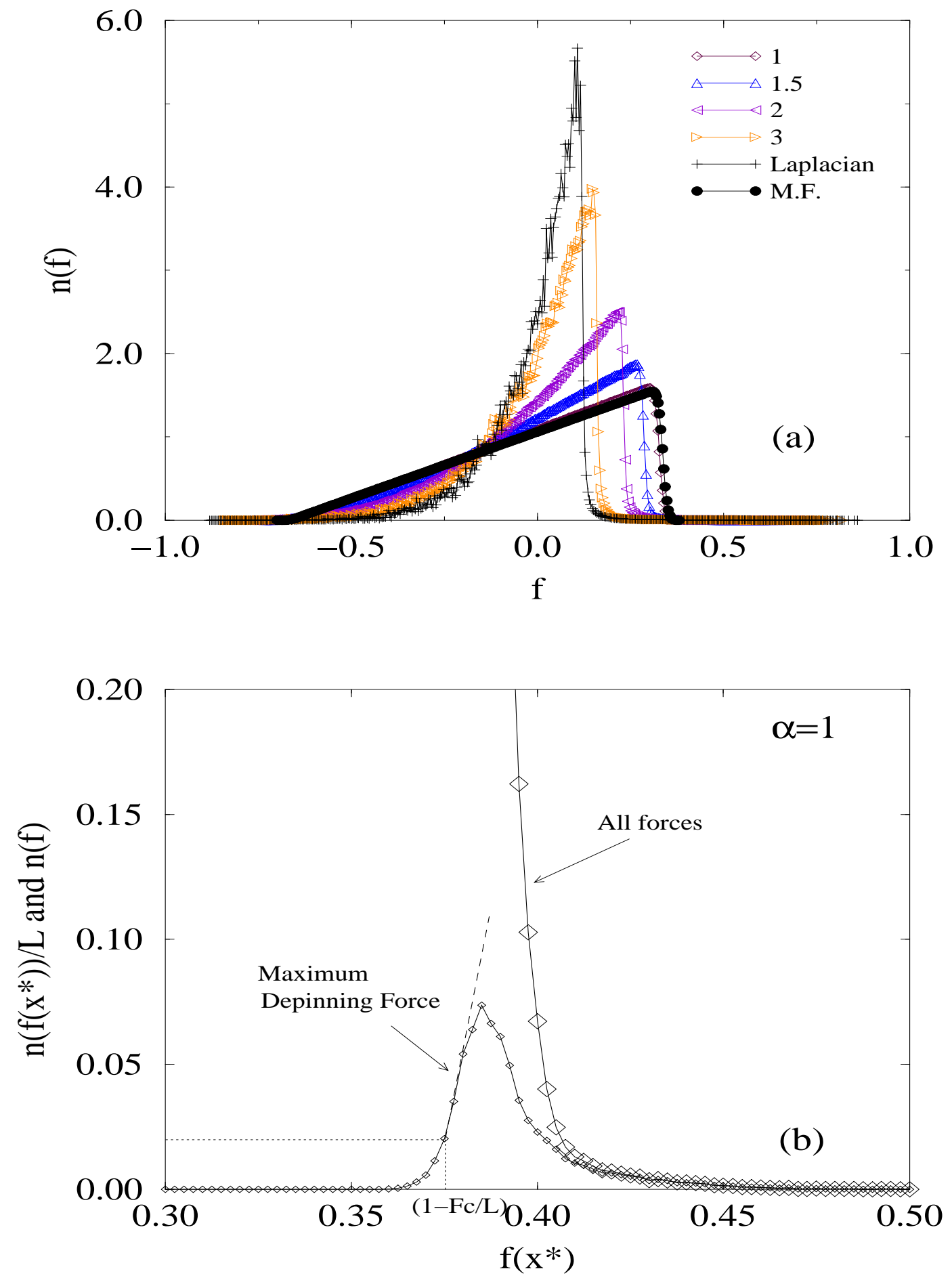

Figure 6 


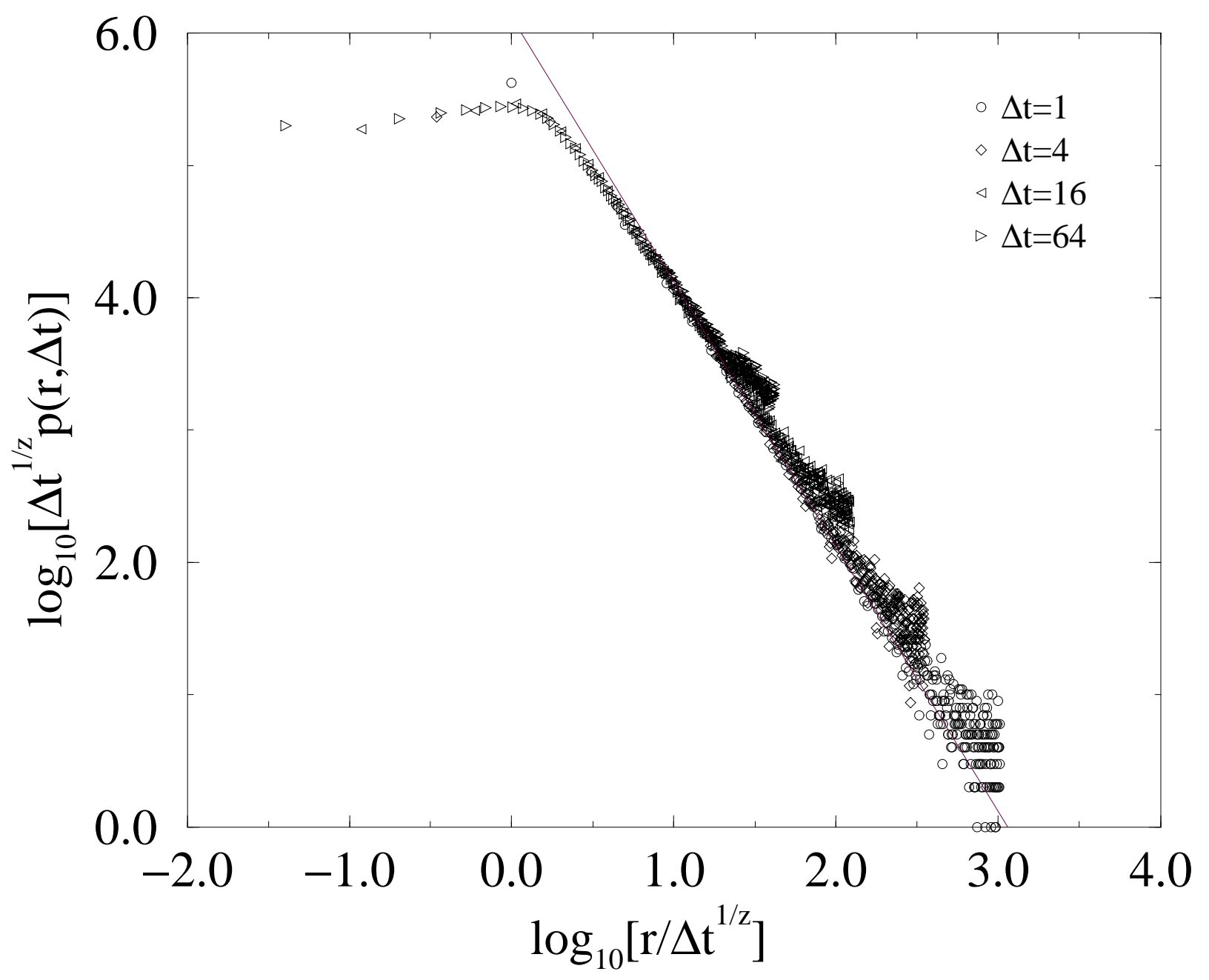

Figure 7 

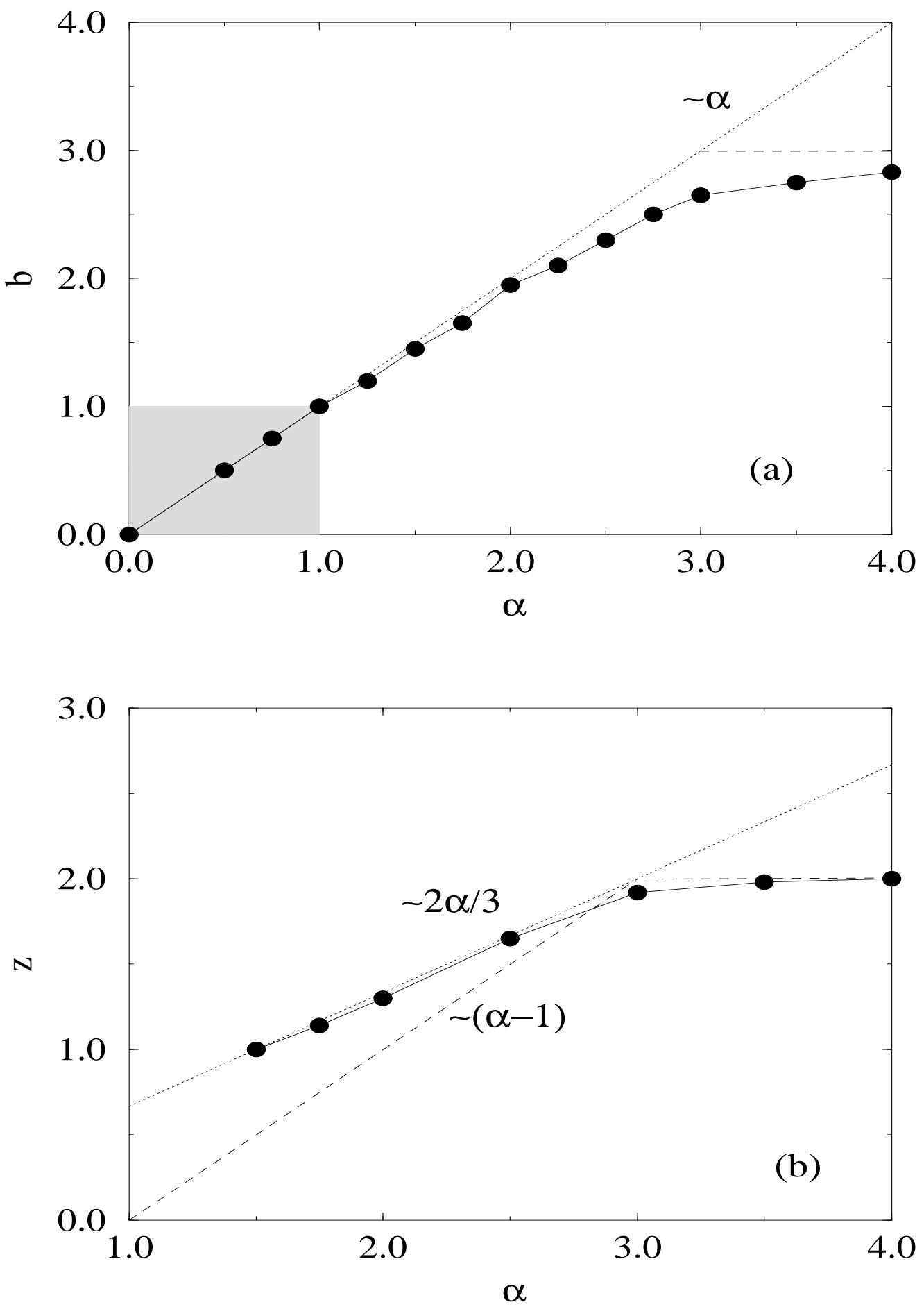

Figure 8 


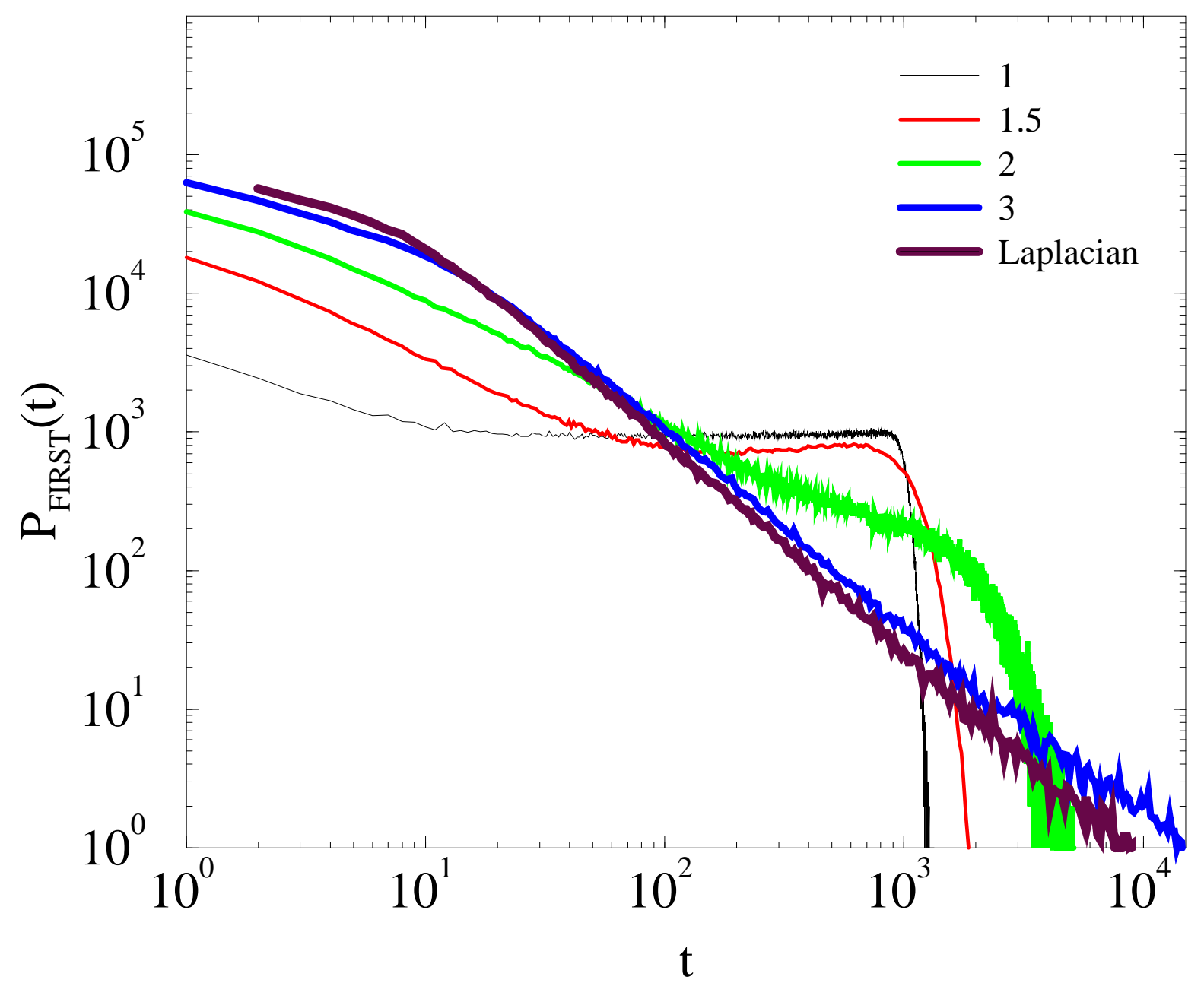

Figure 9 


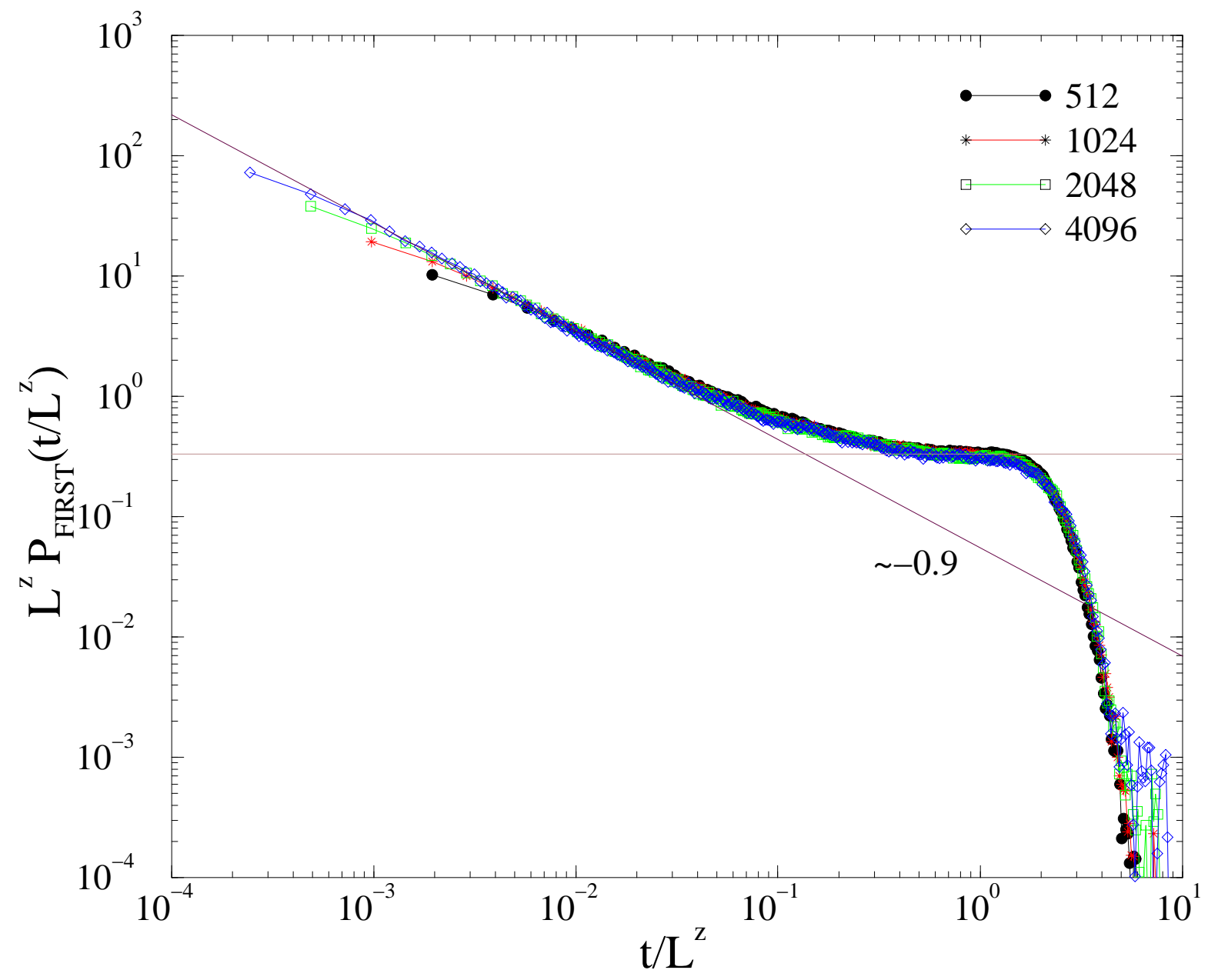

Figure 10 


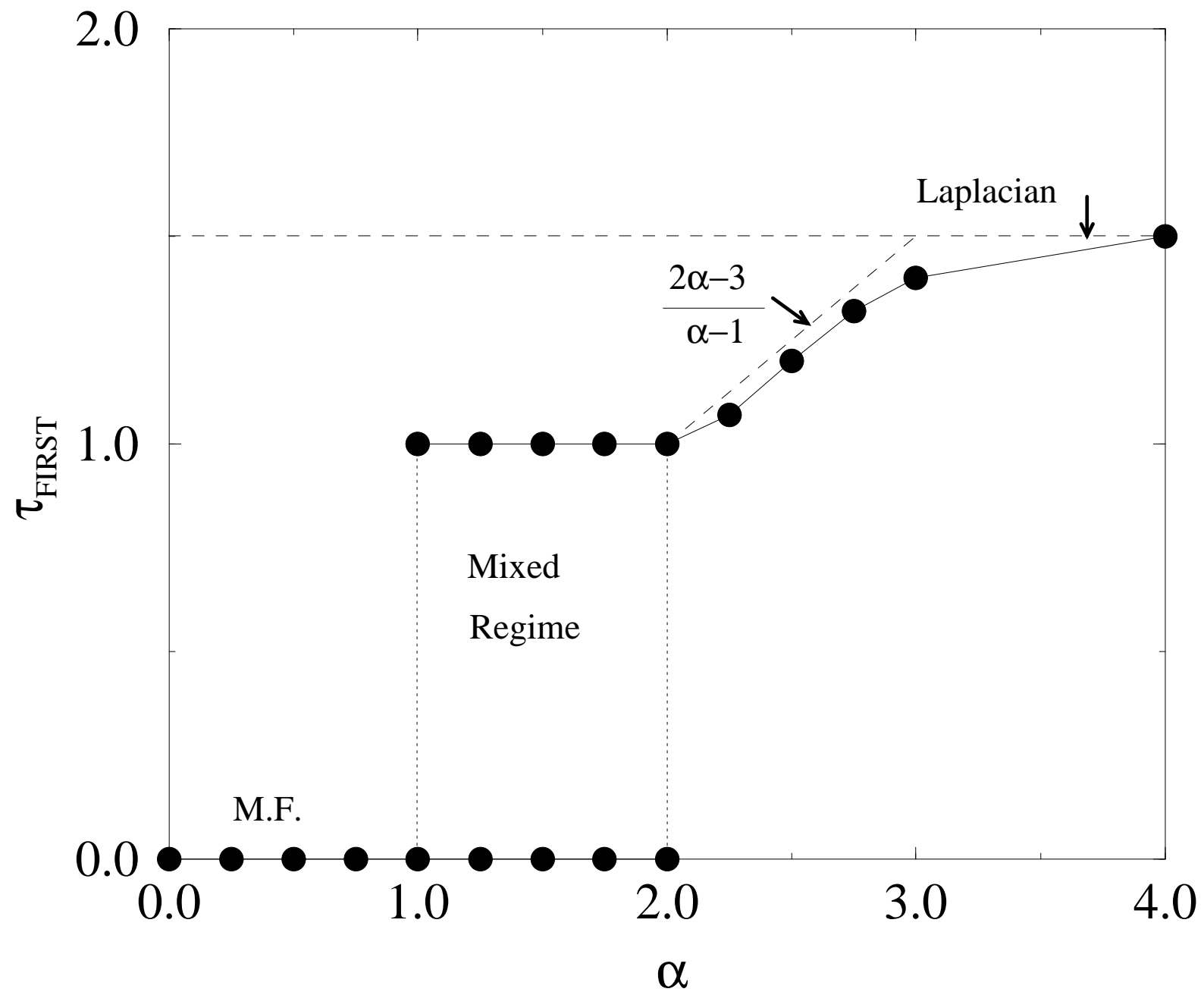

Figure 11 


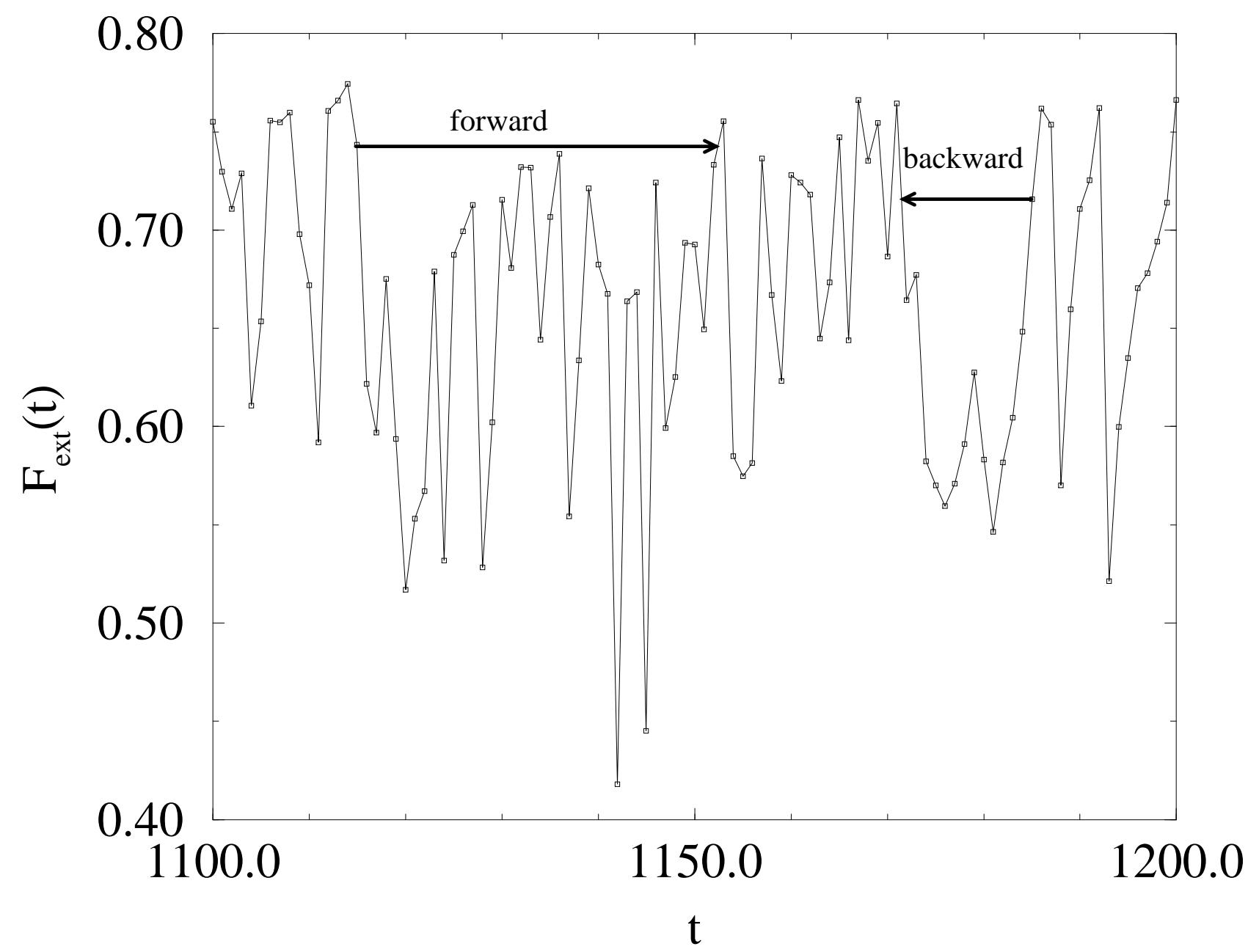

Figure 12 


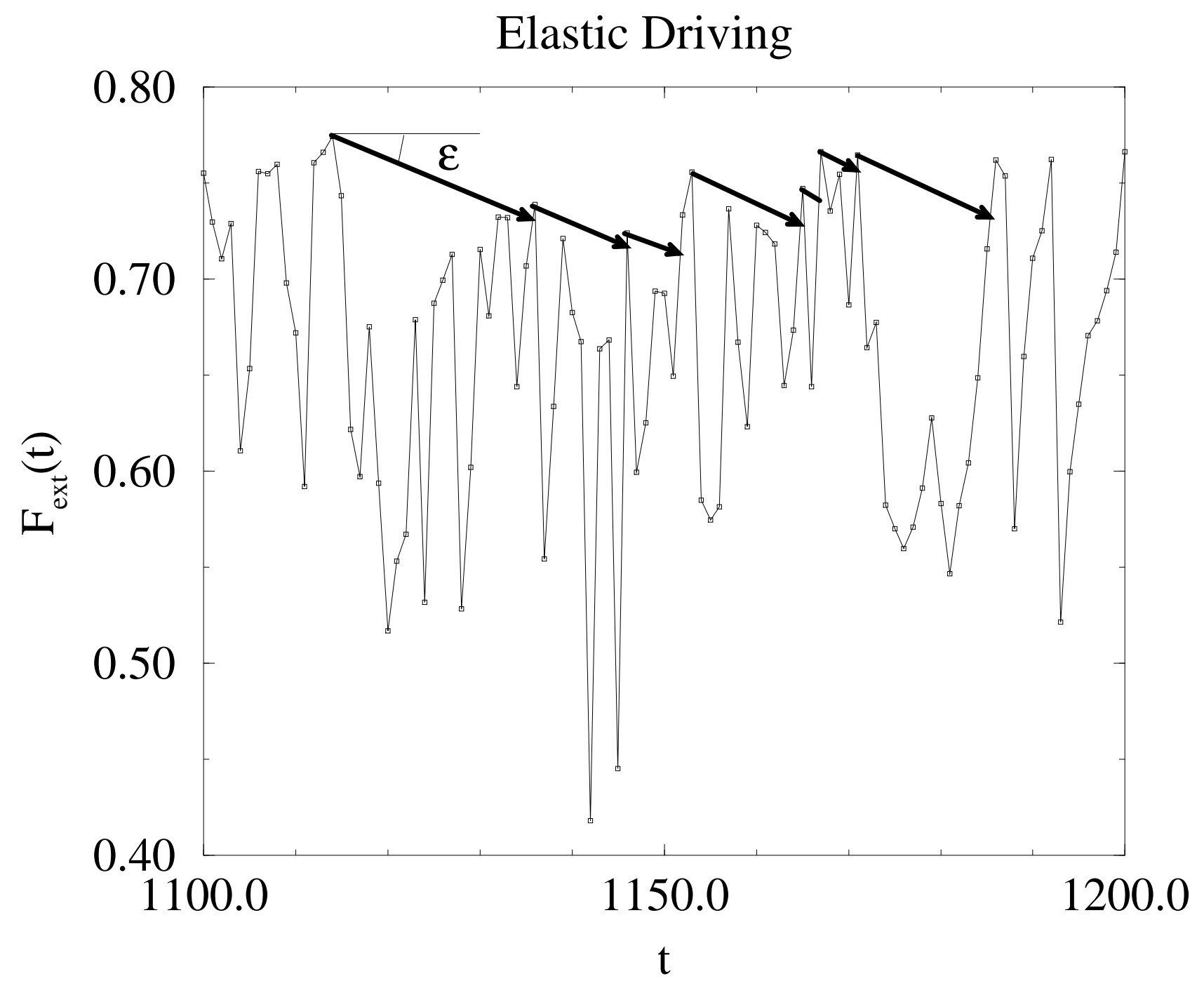

Figure 13 


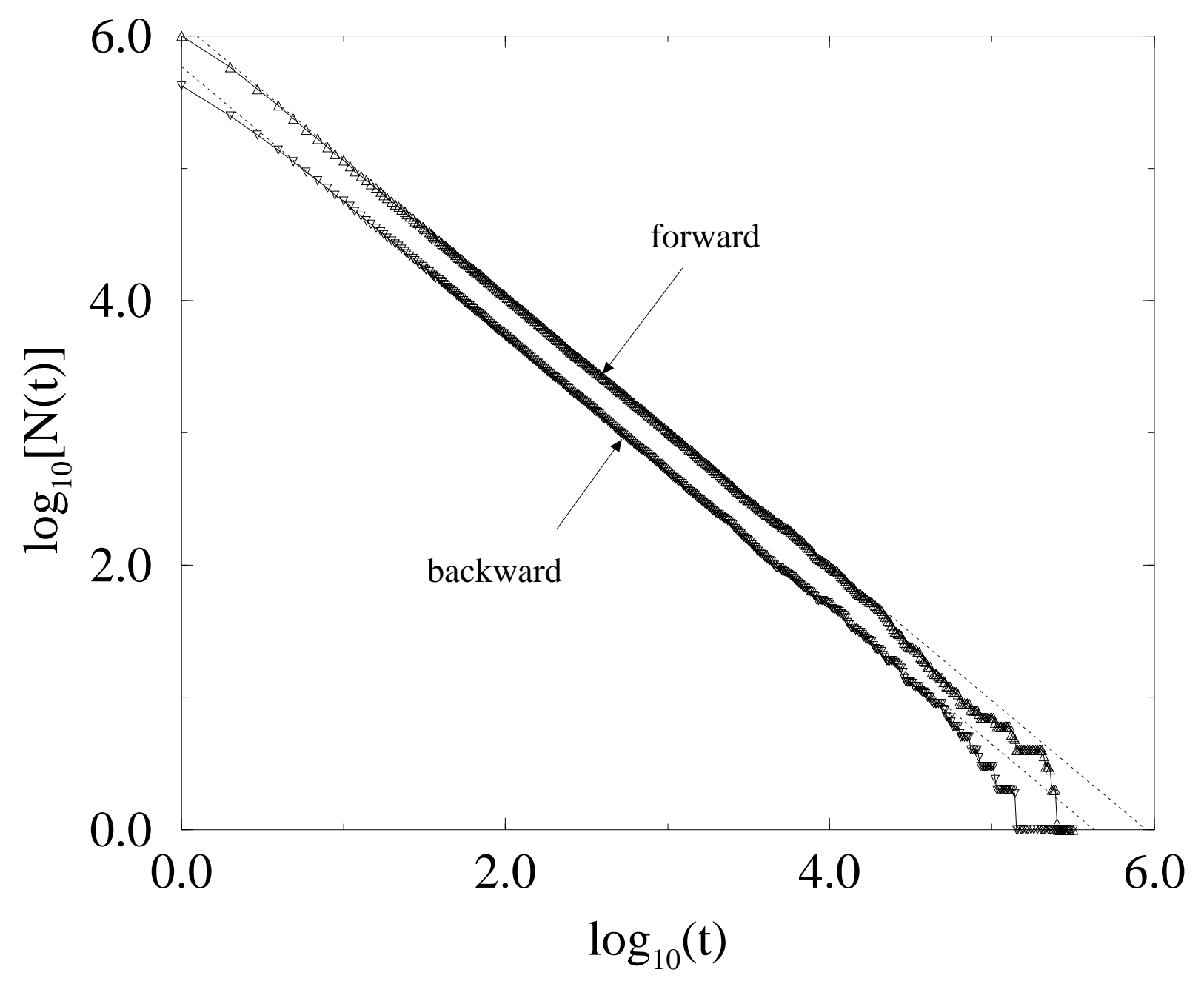

Figure 14 


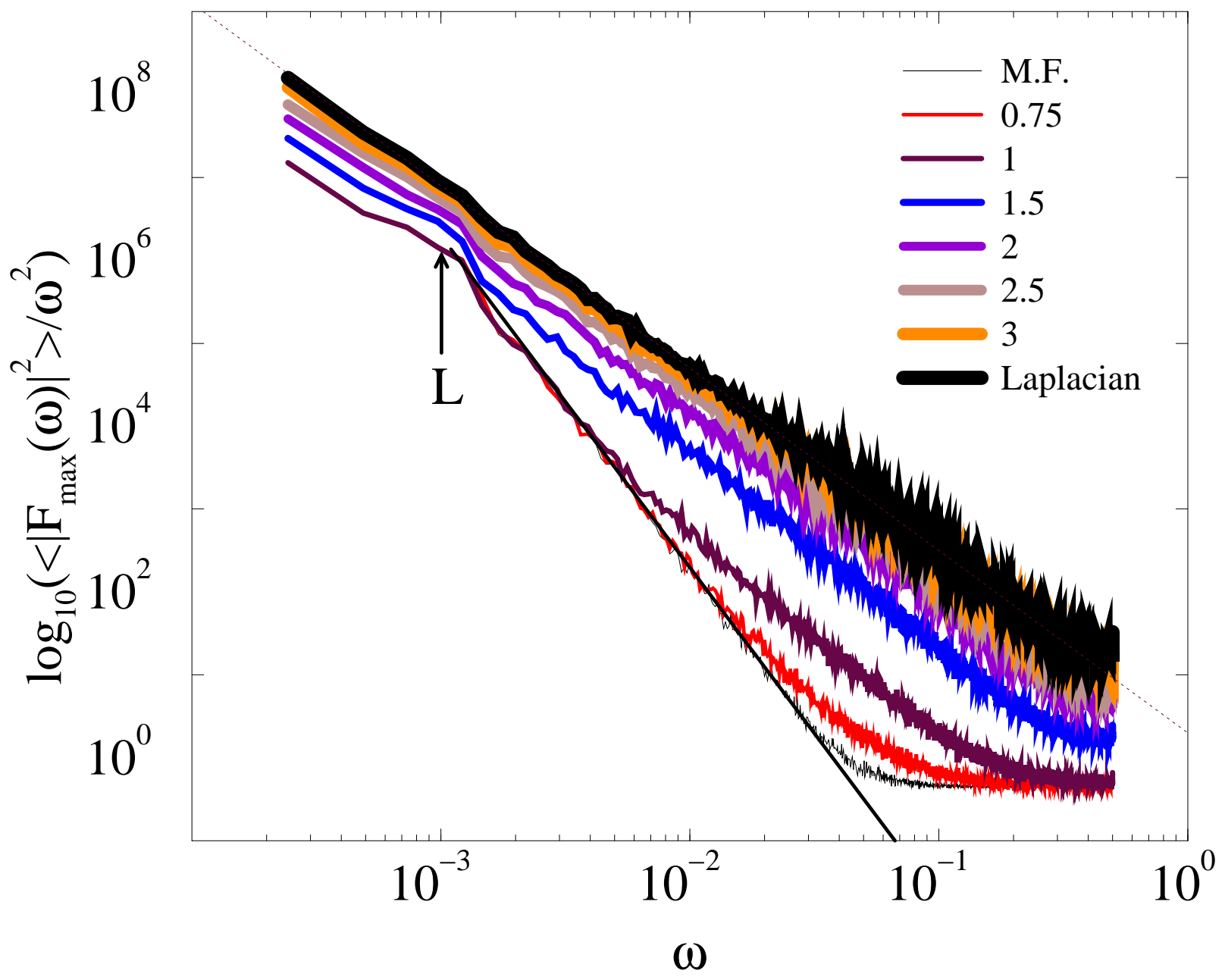

Figure 15 


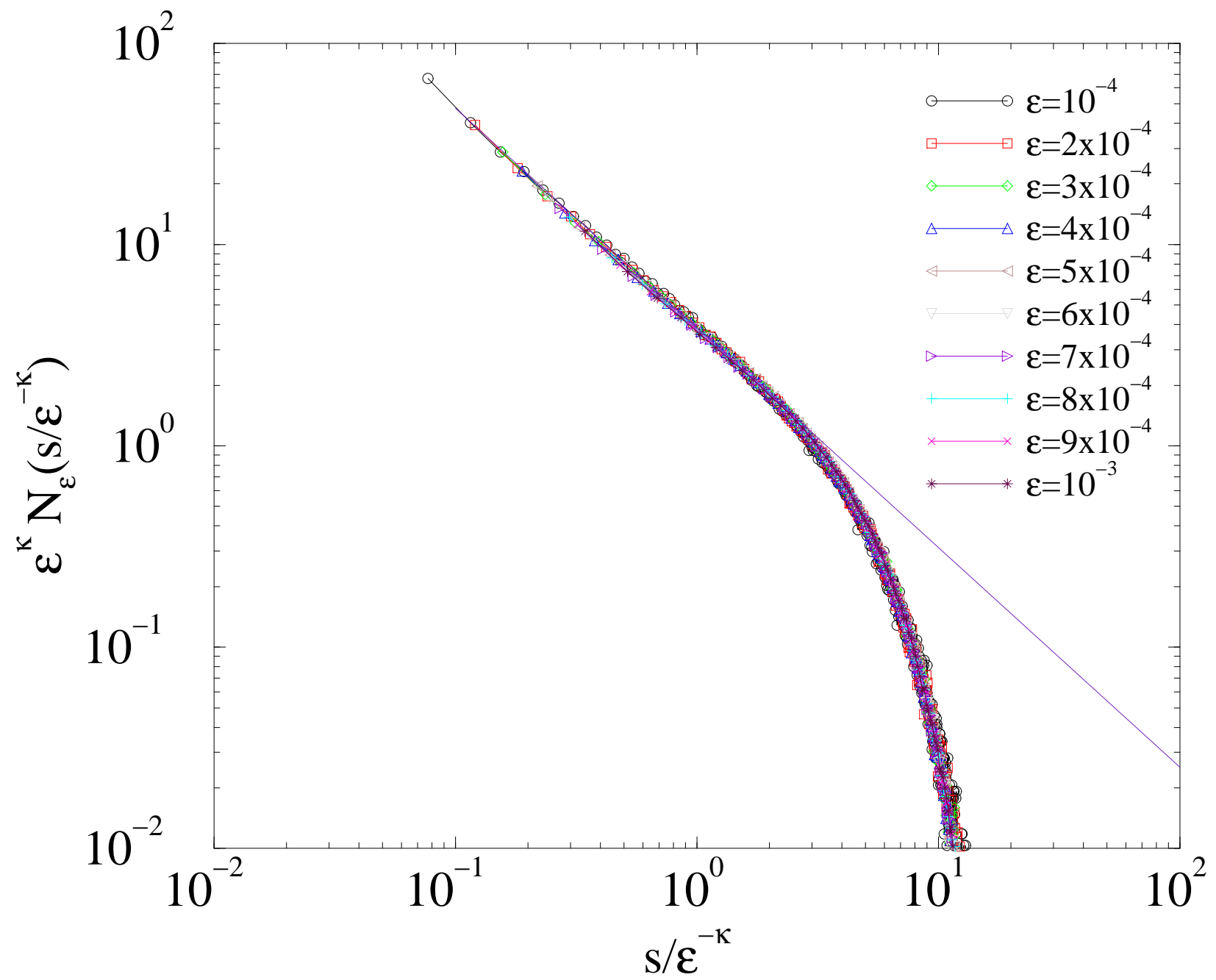

Figure 16 


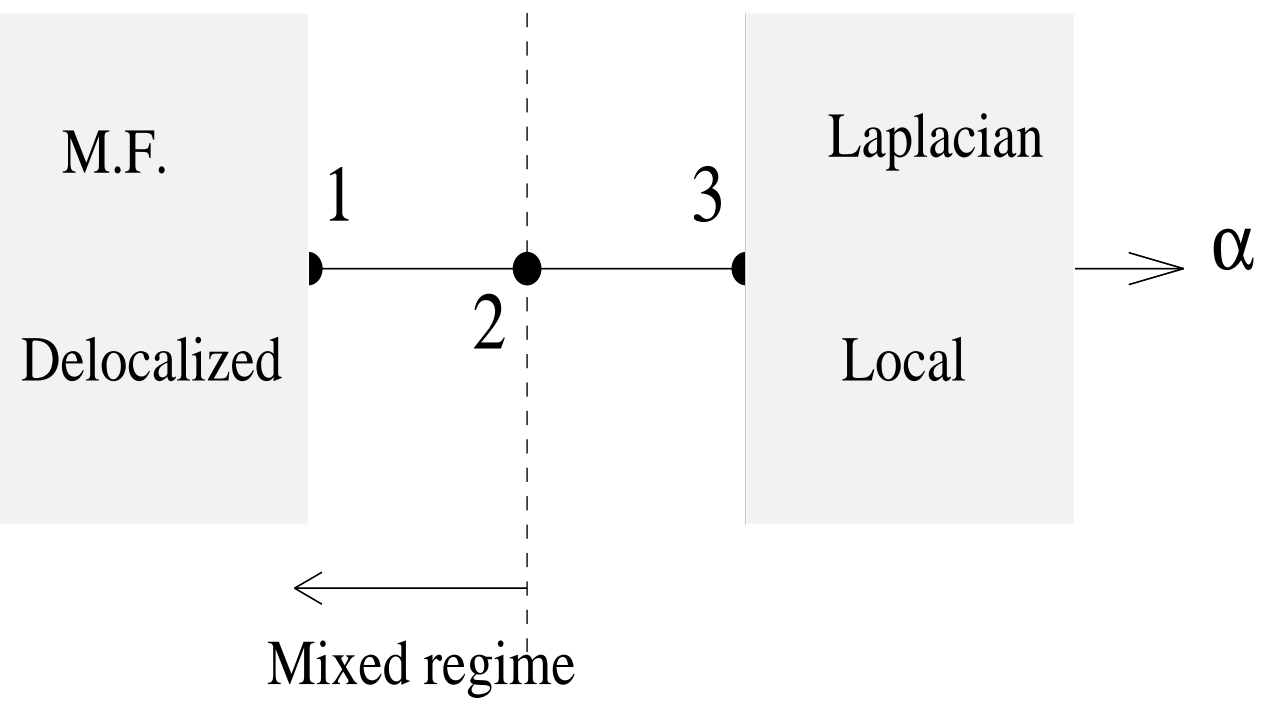

Figure 17 